\title{
APOBEC mutagenesis inhibits breast cancer growth through induction of a $T$ cell- mediated antitumor immune response
}

Ashley V. DiMarco ${ }^{1}$, Xiaodi Qin², Sarah Van Alsten³, Brock McKinney ${ }^{1}$, Nina Marie G. Garcia ${ }^{1}$, Jeremy Force ${ }^{4}$, Brent A. Hanks ${ }^{4}$, Melissa A. Troester ${ }^{3}$, Kouros Owzar ${ }^{2}$, Jichun Xie ${ }^{2}$, James V. Alvarez $^{1}$

${ }^{1}$ Department of Pharmacology and Cancer Biology, Duke University School of Medicine; ${ }^{2}$ Department of Biostatistics and Bioinformatics, Duke University School of Medicine; ${ }^{3}$ Department of Epidemiology, Gillings School of Global Public Health, University of North Carolina at Chapel Hill

${ }^{4}$ Division of Medical Oncology, Department of Medicine, Duke Cancer Institute

Running title: APOBEC activity induces antitumor immunity in breast cancer

Keywords: APOBEC, mutational signatures, APOBEC3B, breast cancer, immunotherapy, checkpoint blockade

The authors declare no potential conflicts of interest. 


\section{ABSTRACT}

The APOBEC family of cytidine deaminases is one of the most common endogenous

3 sources of mutations in human cancer. Genomic studies of tumors have found that APOBEC

4 mutational signatures are particularly enriched in the HER2 subtype of breast cancer and have

5 been associated with immunotherapy response in diverse cancer types. However, the direct

6 consequences of APOBEC mutagenesis on the tumor immune microenvironment have not been

7 thoroughly investigated. To address this, we developed syngeneic murine mammary tumor models

8 with inducible expression of APOBEC3B. We found that APOBEC activity induces an antitumor

9 adaptive immune response and $\mathrm{CD}^{+} \mathrm{T}$ cell-mediated tumor growth inhibition. While polyclonal

10 APOBEC tumors had a moderate growth defect, clonal APOBEC tumors were almost completely

11 rejected by the immune system, suggesting that APOBEC-mediated genetic heterogeneity limits

12 the antitumor adaptive immune response. Consistent with the observed immune infiltration in

13 APOBEC tumors, APOBEC activity sensitized HER2-driven breast tumors to checkpoint

14 inhibition. In human breast cancers, the relationship between APOBEC mutagenesis and

15 immunogenicity varied by breast cancer subtype and the frequency of subclonal mutations. This

16 work provides a mechanistic basis for the sensitivity of APOBEC tumors to checkpoint inhibitors

17 and suggests a rationale for using APOBEC mutational signatures as a biomarker predicting

18 immunotherapy response in HER2-positive breast cancers. 


\section{SIGNIFICANCE}

20 APOBEC mutational signatures are observed in many cancers, yet the consequences of

21 these mutations on the tumor immune microenvironment are not well understood. Using a novel

22 mouse model, we show that APOBEC activity sensitizes HER2-driven mammary tumors to

23 checkpoint inhibition and could inform immunotherapy treatment strategies for HER2-positive

24 breast cancer patients. 


\section{INTRODUCTION}

More than 50 distinct mutational signatures have been identified in cancer genomes (1-3).

27 These signatures are thought to reflect transient or ongoing exogenous and endogenous mutational

28 processes that occur over the lifetime of normal cells and during tumor development. Single-base

29 substitution (SBS) signature 2 is characterized by C-to-T transitions within the trinucleotide motif

30 of TCW (where W represents adenine or thymine), and SBS signature 13 is defined by C-to-G

31 transversions within the same TCW motif. Both signatures are attributed to the APOBEC

32 (apolipoprotein B mRNA editing enzyme, catalytic polypeptide-like) family of cytidine

33 deaminases $(1,2)$. APOBEC enzymes catalyze the deamination of cytosine to uracil on single-

34 stranded DNA which, following repair, manifests predominantly as C-to-T and C-to-G point

35 mutations. The majority of these substitutions are distributed stochastically throughout the somatic

36 genome; however, some are localized in multi-kilobase long, strand-coordinated clusters referred

37 to as 'kataegis' (1,4-6). While APOBEC enzymes have evolutionarily conserved activity in the

38 generation of antibody diversification and restriction of viruses and endogenous retrotransposons,

39 their off-target mutagenic activity on the host somatic genome drives cancer genome instability

40 (reviewed by $(7,8)$ ). APOBEC-mediated mutational signatures have been detected in at least 22

41 different tumor types and are particularly enriched in bladder, head and neck, cervical, and breast

42 cancer $(9,10)$. Importantly, nearly half of breast cancers exhibit kataegis hypermutation clusters

43 (11). Among breast cancer subtypes, human epidermal growth factor receptor 2-positive (HER2 $\left.{ }^{+}\right)$

44 breast tumors are reported to have the highest median levels of APOBEC signature enrichment $45(2,9,12)$.

46 Somatic mutations in cancer can give rise to unique mutant peptides that serve as immune-

47 reactive neoantigens, allowing cytotoxic T cells to target tumor cells for elimination $(13,14)$. Thus, 
recent work has focused on understanding the role of ongoing mutational processes in contributing to tumor immunogenicity and response to immunotherapies (15-17). Despite the prevalence of APOBEC mutational signatures in breast cancer, these tumors are traditionally thought to be

51 poorly immunogenic or "cold". Breast tumors generally have a modest tumor mutation burden

52 (TMB) (2) and low tumor-infiltrating lymphocytes relative to more immunogenic cancers that

53 exhibit robust immune infiltration and are sensitive to checkpoint inhibitors (reviewed by (18-

54 20)). However, the initial trials of anti-PD-1 and anti-PD-L1 monotherapy in triple-negative breast

55 cancer (TNBC) showed promising objective response rates of up to $19 \%(21,22)$. The combination

56 of anti-PD-L1 and nab-paclitaxel had an objective response rate of $39 \%$ and prolonged overall

57 survival, leading to its FDA approval for advanced/metastatic PD-L1+ TNBC in 2019, the first

58 approval of immunotherapy for breast cancer (23). However, checkpoint inhibitor clinical trials

59 have been less successful for $\mathrm{HER}_{2}{ }^{+}$breast cancer patients. In an initial trial for anti-PD-L1

60 monotherapy in metastatic breast cancer, there were no objective responses in the HER2+ subtype

61 (24). When trastuzumab was combined with anti-PD-1 for HER2+ patients, responses ranged from

$620 \%-15.2 \%$ and were highly dependent on PD-L1 status (25). However, APOBEC mutational

63 signatures have yet to be investigated as a specific class of hypermutation that transforms an

64 immunologically "cold" HER2+ breast tumor "hot", rendering the tumor responsive to checkpoint

65 inhibition.

Recent work on how mutational signatures impact tumor immunity has revealed several

67 pieces of evidence potentially implicating APOBEC mutagenesis in immunotherapy response. In

68 pan-cancer analyses from The Cancer Genome Atlas (TCGA), the kataegis-like APOBEC

69 mutational signature was significantly correlated with PD-L1 expression and neopeptide

70 hydrophobicity $(26,27)$. Further, APOBEC signatures were associated with a greater likelihood of 
71 response to immune checkpoint inhibition in non-small cell lung cancer (NSCLC) (28), head and

72 neck cancer, bladder cancer (29), and in a small cohort of breast cancer patients (30). In a recent

73 study using mouse models of TNBC, overexpression of the murine APOBEC3 ortholog sensitized

74 tumors to checkpoint inhibitors (31). Additionally, overexpression of human APOBEC3B in a

75 vaccine setting sensitized mouse melanomas to checkpoint inhibition (32). However, the direct

76 consequences of APOBEC mutagenesis on the tumor immune microenvironment and tumor

77 growth in the absence of checkpoint inhibitors have not been thoroughly explored. A mechanistic

78 understanding of how APOBEC mutagenesis alters the tumor immune microenvironment would

79 inform the use of immune therapies for human tumors with APOBEC mutational signatures.

80 Furthermore, despite the high enrichment of APOBEC signatures in HER2+ breast cancer, no

81 studies to our knowledge have investigated a role for APOBEC mutagenesis in conferring clinical

82 benefit to checkpoint blockade in HER2+ breast cancer.

83 To address these questions, we developed a syngeneic, immunocompetent murine HER2-

84 driven mammary tumor model with APOBEC activity. Using this model, we examined the

85 consequences of APOBEC activity and genetic heterogeneity on tumor growth, investigated

86 tumor-immune system interactions in APOBEC tumors, and assessed the therapeutic response of

87 these tumors to checkpoint inhibitor therapy. Finally, we examined the relationship between

88 APOBEC mutagenesis and adaptive immune response in human breast tumors.

\section{RESULTS}

91 Ectopic expression of A3B in murine mammary tumor cells is not lethal and induces cytidine

92 deaminase activity 
To induce APOBEC mutagenesis in vivo in an immunocompetent HER2-driven mammary

94 tumor model, we utilized the SMF cell line, which is derived from a mammary tumor arising in

95 the MMTV-Neu/Her2 mouse model on the FVB background (33). We engineered SMF cells to

96 conditionally express the human APOBEC family member, APOBEC3B (A3B), and thereby

97 acquire APOBEC mutational signatures during tumor progression. Along with APOBEC3A, A3B

98 is one of the major contributors of APOBEC mutations in cancer genomes $(3,10,34,35)$. Studies

99 in yeast and mammalian cells have shown that expression of A3B is sufficient to induce a kataegis-

100 like pattern, and preferentially induce mutations at the TCW trinucleotide context resembling SBS

101 signatures 2 and 13 in cancer genomes (5,36,37), whereas the murine APOBEC3 ortholog localizes

102 to the cytoplasm and has low catalytic activity (38). The SMF cell line was stably transduced with

103 a lentivirus encoding reverse tetracycline-controlled transactivator (rtTA) and a lentivirus

104 encoding rtTA-responsive human A3B (referred to as "SMF-A3B cells"). This system allows for

105 titratable and reversible expression of A3B in tumor cells with the administration of doxycycline

106 (dox) in the cell culture medium or in the drinking water of mice.

107 To characterize the A3B expression system in vitro, SMF-A3B cells were cultured with 108 increasing concentrations of dox, with or without subsequent removal of dox from the medium.

109 A3B mRNA and protein expression were dose-responsive and reversible (Fig. 1A, B).

110 Additionally, A3B protein was constitutively localized to the nucleus in the presence of dox,

111 demonstrating proper subcellular localization of this APOBEC family member (39)(Fig. 1C). In

112 an in vitro cytidine deaminase activity assay, increasing concentrations of dox induced dose-

113 responsive deaminase activity in SMF-A3B cells (Fig. 1D). A3B expression did not affect cell

114 proliferation or survival, as measured by an ATP-based cell viability assay and colony formation

115 assay (Fig. 1E-G). For subsequent experiments we used a dox concentration $(1 \mu \mathrm{g} / \mathrm{mL})$ that 
116 induced A3B expression levels and deaminase activity levels comparable to that of the APOBEC-

117 high human HER2+ breast cancer cell line, BT474 (Fig. 1A, D). These data suggest SMF-A3B is

118 a suitable system to induce A3B expression and cytidine deaminase activity in a syngeneic,

119 orthoptic murine tumor model.

A3B expression does not affect tumor growth in immunodeficient mice and is predicted to induce APOBEC-mediated mutational signatures

We first tested whether expression of A3B affects tumor growth in the absence of an

124 adaptive immune system. SMF-A3B cells were implanted in the mammary fat pad of 125 immunodeficient NOD.Cg-Prkdc $c^{\text {scid }} I l 2 \mathrm{rg}^{\mathrm{tm} / \mathrm{Wjl}} / \mathrm{SzJ}$ (NSG) mice, and mice received either normal 126 or dox drinking water throughout the duration of tumor growth to express A3B. Control and A3B127 expressing tumors grew at similar rates (Supplementary Fig. S1A), indicating that in the absence 128 of a functional immune system, A3B expression does not affect tumor growth. This is consistent with the finding that $\mathrm{A} 3 \mathrm{~B}$ expression does not affect the growth or viability of SMF cells in vitro.

We next examined whether SMF-A3B tumors have evidence of APOBEC mutagenesis.

131 Because APOBEC-induced mutations are randomly distributed throughout the genome, it is

132 technically difficult to detect these mutations in a heterogeneous population of cancer cells $(36,40)$.

133 Therefore, to measure the APOBEC mutational process in mouse tumors generated from the SMF-

134 A3B cell line, we developed a gene expression-based classifier for prediction of APOBEC 135 mutational signatures. The classifier was trained using sets of differentially expressed genes from 136 RNA-seq data of APOBEC-high and APOBEC-low breast cancers from TCGA using 10-fold 137 cross validation (as determined by APOBEC mutational signature enrichment score from whole138 exome sequencing; see Methods). This analysis suggested that a 10-gene classifier was optimal 
139 for prediction. The genes selected for the classifier were $A X I N 2, C C D C 157, I C O S, N A G S, N X P H 3$, PRODH, PSD, SRRM3, STMN3, and TTC25.

We next used this classifier to test whether SMF-A3B tumors in NSG mice have evidence

142 of APOBEC mutagenesis. We performed RNA-seq on 6 control tumors and 6 tumors expressing

143 A3B. When applied to this independent dataset, the classifier correctly identified 4 of 6 A3B-

144 expressing tumors as well as 4 of 6 control tumors $(66 \%$ sensitivity and $66 \%$ specificity,

145 Supplementary Fig. S1B), for an overall accuracy in mouse tumors of $66 \%$. This indicates that the

146 gene expression-based classifier may be used to predict APOBEC mutational signatures in the

147 genomes of human and murine tumors, and A3B-expressing tumors generated from SMF-A3B

148 cells are likely to harbor genomic APOBEC-mediated mutations.

APOBEC activity slows mammary tumor growth and triggers the infiltration of antitumor

\section{adaptive immune cells}

Given the evidence that the APOBEC mutational signature is associated with both an immune response and sensitivity to immunotherapy in NSCLC, bladder, and head and neck cancer,

154 we examined the effects of in vivo APOBEC activity on the tumor immune microenvironment.

155 SMF-A3B cells were orthotopically implanted bilaterally in the mammary gland of syngeneic,

156 immunocompetent wildtype FVB mice. One cohort of mice was administered dox in the drinking

157 water to induce A3B expression and APOBEC activity in the tumor cells throughout tumor growth

158 ("APOBEC tumors"), while the control cohort received normal drinking water (Fig. 2A).

159 Interestingly, APOBEC tumors grew significantly slower than control tumors and had a smaller

160 mass at endpoint (Fig. 2B). Immunofluorescence staining of APOBEC and control tumors for a

161 marker of double-stranded DNA breaks, $\gamma \mathrm{H} 2 \mathrm{AX}$, showed no activation of the DNA damage 
response in vivo (Supplementary Fig. S1C, D). Similarly, A3B expression for two weeks did not induce $\gamma \mathrm{H} 2 \mathrm{AX}$ or cleaved PARP in SMF-A3B cells in vitro (Supplementary Fig. S1E). Taken together with the finding that A3B expression does not affect cell growth in vitro (Fig. 1E-G) or tumor growth in immunodeficient NSG mice (Supplementary Fig. S1A), this suggests that the growth defect of APOBEC tumors was mediated by a tumor cell-extrinsic mechanism, specifically

167 the immune response.

To gain insight into how A3B expression alters the tumor microenvironment (TME) of APOBEC tumors, six mice per cohort were randomly selected for immune profiling by flow cytometry (see Supplementary Fig. S2 for gating strategy and Supplementary Fig. S3 for

171 representative FACS plots). APOBEC tumors showed a substantial infiltration of total leukocytes $172\left(\mathrm{CD}^{+} 5^{+} \mathrm{EpCAM}^{-}\right)$compared to control tumors (Fig. 2D). $\mathrm{CD}^{+}$T cells $\left(\mathrm{CD} 45^{+} \mathrm{CD} 3^{+} \mathrm{CD}^{+}\right), \mathrm{CD}^{+}$

$173 \mathrm{~T}$ cells $\left(\mathrm{CD}^{4} 5^{+} \mathrm{CD}^{+} \mathrm{CD}^{+}\right)$, and $\mathrm{CD} 103^{+}$dendritic cells (DCs; CD45+CD11 ${ }^{+} \mathrm{MHC}-\mathrm{II}^{+} \mathrm{F} 4 / 80^{-}$

$174 \mathrm{CD}_{103^{+}}$) were expanded in the APOBEC TME, as measured both as the percentage of CD45 ${ }^{+}$cells

175 (Fig. 2C) and the percentage of total live cells (Fig. 2D). There was no change in the infiltration

176 of natural killer $(\mathrm{NK})$ cells $\left(\mathrm{CD} 45^{+} \mathrm{NK} 1.1^{+} \mathrm{CD} 3^{-}\right)$, although several subsets of cells that may have

177 immunosuppressive potential were significantly reduced in the APOBEC tumors, including the

178 fraction of $\mathrm{T}$ regulatory cells (Tregs; $\mathrm{CD}^{4} 5^{+} \mathrm{CD} 3{ }^{+} \mathrm{CD} 4{ }^{+} \mathrm{FOXP} 3^{+}$), type- $2 \mathrm{~T}$ helper cells (Th2;

$\left.179 \mathrm{CD} 5^{+} \mathrm{CD}^{+} \mathrm{CD}^{+} \mathrm{GATA}^{+}\right)$, and tumor-associated macrophages $\left(\mathrm{CD} 45^{+} \mathrm{F} 4 / 80^{+} \mathrm{CD} 11 \mathrm{c}^{\text {low }}\right)($ Fig.

180 2D). Furthermore, APOBEC tumors were comprised of more immune cells producing the

181 proinflammatory cytokine interferon- $\gamma\left(\operatorname{IFN} \gamma ; \mathrm{CD}_{4} 5^{+} \mathrm{IFN} \gamma^{+}\right)$, and cytotoxic granule granzyme B

182 (GZMB; CD45+GzmB $\left.{ }^{+}\right)($Fig. 2E, F). Finally, PD-1+ immune cells (CD45+PD-1+ $)$and PD-L1 ${ }^{+}$

183 tumor cells (EpCAM+PD-L1+) were elevated in the APOBEC tumors compared to control tumors, 184 suggesting an active $\mathrm{T}$ cell-mediated immune response and potential feedback signaling leading 
to T cell dysfunction (Fig. 2E, F). A similar expansion of CD8 ${ }^{+} \mathrm{T}$ cell and CD103+ DC populations

186 was observed in the tumor-draining lymph nodes from mice with APOBEC tumors

187 (Supplementary Fig. S4A). The defect in APOBEC tumor growth and enhanced immune

188 infiltration measured by flow cytometry was also observed in an independent experiment

189 (Supplementary Fig. S4B, C). To extend these results to a second breast cancer cell line, we

190 engineered inducible A3B expression in the mouse breast cancer line, EMT6, (Supplementary Fig.

191 S4D-F). As with SMF tumors, EMT6 APOBEC tumors grew more slowly and had increased

192 infiltration of leukocytes when implanted in syngeneic BALB/c mice (Supplementary Fig. S4G-

193 I).

Next, to examine the relationship between immune infiltration and tumor size, we 195 measured the correlation between immune cell abundance and tumor size at endpoint in SMF 196 tumors. $\mathrm{CD}^{+} \mathrm{T}$ cells, $\mathrm{CD} 4^{+} \mathrm{T}$ cells, $\mathrm{CD} 103^{+} \mathrm{DCs}$, and IFN $\gamma^{+}$cells were each negatively correlated 197 with tumor size (Fig. 2G). This suggests that the adaptive immune response may mediate the growth defect observed in APOBEC tumors.

The localization of $\mathrm{T}$ cells in the TME is an important factor that influences tumor immunity and responses to immunotherapy (reviewed by (43)). T cells can be excluded from the

201 tumor core and instead localize to the periphery in murine models and human tumors (44-47), and

202 this exclusion may be one mechanism of immune suppression. Therefore, to assess $\mathrm{T}$ cell

203 localization in APOBEC tumors, we performed immunohistochemical (IHC) staining for CD3 on

204 an independent cohort of SMF tumors. CD3 staining was consistent with flow cytometry analyses

205 and revealed an increase in the total number of T cells in APOBEC tumors. The T cells were most

206 concentrated on the periphery of the APOBEC tumors, although importantly, significant levels of

207 T cells also infiltrated the tumor core (Fig. 2H, I). 
The growth defect of APOBEC tumors is dependent on the A3B catalytic activity, not A3B

214 directed mutagenesis of one of the A3B catalytic domains (E255Q). SMF cells were transduced

215 with lentivirus expressing the A3B catalytic mutant to generate SMF-A3B inactive cells. Dox treatment led to expression of catalytically-dead A3B in these cells, but there was no detectable

217 increase in deaminase activity (Supplementary Fig. S5A-C). SMF-A3B inactive cells were then 218 injected into the mammary glands of immunocompetent wildtype mice on dox water to induce expression of the full-length, catalytically dead A3B protein. Tumors expressing catalytically dead tumors was due to A3B-mediated mutagenesis, as opposed to the expression of A3B protein, we took advantage of the reversibility of the dox-inducible system. SMF-A3B cells were cultured with

227 dox in the cell medium for two weeks to mutagenize the cells and then dox was removed to 228 downregulate A3B expression. These in vitro APOBEC mutagenized cells retain A3B-catalyzed 229 mutations but do not express A3B protein. The proliferation rate of in vitro APOBEC mutagenized 230 cells was similar to control, non-mutagenized cells (Supplementary Fig. S5F). In contrast, when 
231 implanted into the mammary gland of wildtype mice without dox in their drinking water

232 (Supplementary Fig. S5G), the in vitro APOBEC mutagenized tumors grew more slowly than

233 control tumors and had evidence of an increased adaptive immune response, as measured by qRT-

234 PCR for T cell-specific genes Gzma, Prf-1, Tbx21 (Supplementary Fig. S5H, I). The growth defect

235 of in vitro APOBEC mutagenized tumors was not evident in NSG mice (Supplementary Fig. S5J),

236 further confirming the role of the adaptive immune response in mediating the growth defect of

237 APOBEC tumors. Together these data reveal that A3B activity promotes an infiltrated-inflamed

238 TME in HER2-driven murine tumors and leads to an immune-dependent growth defect.

APOBEC activity slows breast tumor growth by stimulating a tumor antigen-specific

\section{adaptive immune response}

To understand the basis of the immune-mediated growth defect of APOBEC tumors, we

243 performed RNA-sequencing on control and APOBEC tumors from either immunocompetent

244 wildtype mice or immunodeficient NSG mice. APOBEC tumors in wildtype mice showed a

245 significant upregulation of adaptive immune response gene ontology (GO) terms, including

246 regulation of $\mathrm{T}$ cell mediated immunity/cytotoxicity/differentiation, antigen processing and

247 presentation, and B cell activation (Fig. 3A, Supplementary Fig. S6A). Moreover, the top two

248 pathways enriched in the APOBEC tumors in wildtype mice by gene set enrichment analysis

249 (GSEA) were allograft rejection (Supplementary Fig. S6B) and IFN $\gamma$ response (Fig. 3B),

250 suggesting an adaptive immune response mechanism of tumor cell killing. In APOBEC tumors

251 harvested from immunodeficient NSG mice, in contrast, the DNA repair pathway was significantly

252 enriched by GSEA (Fig. 3B, Supplementary Fig. S6C), possibly due to the activation of repair

253 pathways following the generation of A3B-catalyzed uracil lesions in the genome. 
255 next interested in studying tumor-specific antigen responses in APOBEC tumors. To gain insight

256 into these responses, we assessed changes in the T cell repertoire between control and APOBEC

257 tumors using T cell receptor (TCR)-sequencing. RNA was extracted from control and APOBEC

258 tumors growing in wildtype mice and used for TCR library preparation and sequencing of the $\beta$

259 chain. The CDR3 variants were interrogated and unique clonotypes were counted. APOBEC

260 tumors had more unique TCR clonotypes than control tumors (Fig. 3C, D). Using the Shannon

261 entropy diversity index to measure the diversity richness of the clonotypes in the population, we

262 found that APOBEC tumors had a higher clonotype diversity than control tumors (Fig. 3E).

263 Finally, we used the diversity evenness $50\left(\mathrm{DE}_{50}\right)$ ratio, which is a measure of the number of

264 clonotypes making up the top $50 \%$ of reads relative to the total number of reads, to assess clonotype

265 evenness. A high $\mathrm{DE}_{50}$ ratio indicates that clonotypes are evenly represented in the population,

266 whereas a low $\mathrm{DE}_{50}$ ratio corresponds to a TCR repertoire that is dominated by specific CDR3

267 clonotypes. This analysis indicated that APOBEC tumors had a lower $\mathrm{DE}_{50}$ ratio than control

268 tumors (Fig. 3F). Taken together, these analyses indicate that the TCR repertoire of APOBEC

269 tumors exhibit increased diversity richness but decreased diversity evenness; interestingly, this

270 pattern has been associated with productive $\mathrm{T}$ cell responses with antitumor effects and successful

271 treatment with immunotherapy (48).

273 tumor bearing mice and co-culturing the cells with autologous tumor cell lysate for 48 hours. Re-

274 stimulation responses were measured by IFN $\gamma$ ELISpot. Autologous APOBEC tumor lysate was

275 capable of re-stimulating splenocytes from APOBEC-tumor bearing mice to produce IFN $\gamma$ at

276 comparable levels to that of naïve splenocytes (NS) stimulated with model antigen, concanavalin 
A (ConA; Fig. 3G). Thus, A3B-mediated mutagenesis may lead to the generation of tumor-specific

278 antigens which are targeted by $\mathrm{T}$ cells.

$\mathrm{CD4}^{+} \mathrm{T}$ cells are required for the tumor growth defect of APOBEC tumors simultaneously (Supplementary Fig. S7E-G). In the absence of both $\mathrm{CD}^{+}$and $\mathrm{CD} 8^{+} \mathrm{T}$ cells, the growth in APOBEC tumors.

APOBEC activity renders murine HER2-driven breast tumors responsive to immune 
inhibition. SMF-A3B cells were implanted in the mammary glands of wildtype mice and mice were administered dox water or control water. When control and APOBEC tumors reached $5 \mathrm{~mm}$ in diameter, mice were treated with combination anti-PD-1 and anti-CTLA-4 therapy twice weekly. Control tumors did not benefit from checkpoint inhibition, consistent with the clinical observation that checkpoint inhibition is not been effective in HER2+ breast cancer patients. In contrast, APOBEC tumor growth was significantly blunted upon treatment with anti-PD-1/antiCTLA4 therapy (Fig. 4A). We defined a complete response (CR) as a full tumor regression (-100\% change in tumor volume from the treatment start) and a partial response (PR) as any reduction in tumor volume from the treatment start. Checkpoint inhibitor treatment led to a partial response in only 1 of the 13 control tumors (Fig. 4B, Supplementary Fig. S8A). In contrast, 7 out of 11 APOBEC tumors had a complete or partial response to combination checkpoint inhibition (Fig. anti-PD-1 monotherapy (Supplementary Fig. S8B). These results show that APOBEC activity sensitized HER2-driven murine breast cancers to combination anti-PD-1/anti-CTLA-4 checkpoint

314 inhibition, but not single agent therapy.

APOBEC-mediated genetic heterogeneity permits immune escape, while clonal APOBEC

\section{tumors remain in cancer-immune equilibrium} cancer heterogeneity and evolution (49-51). In melanoma and NSCLC, mutational and neoantigen

320 heterogeneity reduces antitumor immunity (52-54) and response to checkpoint inhibitor therapy.

321 For instance, lung tumors with more clonal neoantigens are better controlled by neoantigen-

322 specific $\mathrm{T}$ cells and have improved responses to checkpoint inhibitors (55). The role of intratumor 
323 diversity in breast cancer immunogenicity has yet to be thoroughly studied. Thus, we were

324 interested in understanding the consequences of APOBEC-mediated genetic heterogeneity on

325 antitumor immunity and mammary tumor growth in immunocompetent mice.

326 To assess differences between heterogenous and clonal APOBEC tumors, SMF-A3B cells

327 were cultured with dox for 2 weeks to mutagenize and induce genetic heterogeneity in the

328 population of cells, and then dox was removed to downregulate A3B. These cells are referred to

329 as "parental APOBEC", whereas the control, non-mutagenized cells are referred to as "parental

330 control". We next derived single-cell clones by limiting dilution from the parental APOBEC and

331 parental control populations. We screened several clonal populations for the ability to grow at the

332 same rate as parental populations in vitro. Control clone 1 and APOBEC clone 1 grew slower than

333 the parentals, while control clone 2 and APOBEC clone 2 grew at the same rate as parentals (Fig.

334 5A). When we implanted the clones in immunocompromised, athymic nude mice and measured

335 tumor growth, only control clone 2 and APOBEC clone 2 were able to form tumors similarly to

336 the parental counterparts (Fig. 5B). Therefore, we proceeded to study the tumor growth of control

337 clone 2 and APOBEC clone 2 in immunocompetent, wildtype mice. While control clone 2 grew

338 similarly to the parentals, APOBEC clone 2 cells gave rise to very small tumors that remained in

339 a cancer-immune equilibrium until the animals were sacrificed (Fig. 5C). When we compared the

340 average size of tumors formed in the presence or absence of the adaptive immune response, we

341 found tumors formed from APOBEC clone 2 were significantly smaller in wildtype mice (Fig.

342 5D). Thus, APOBEC-mediated heterogeneity may limit the potential of a fully productive immune

343 response against hypermutated breast tumors. In contrast, clonal APOBEC tumor growth may be

344 controlled in cancer-immune equilibrium. 
The APOBEC mutational signature is associated with an adaptive immune response in basal-

347

348

349

350

351

352

353

354

355

356

357

358

359

360

361

362

363

364

365

366

367

\section{like but not HER2-enriched human breast cancers}

We were next interested in determining whether human breast tumors with APOBEC mutagenesis have evidence of an increased adaptive immune response. To do this, we analyzed breast tumors from TCGA for which both whole-exome sequencing (WES) and RNA-sequencing data were available. To assess the enrichment of APOBEC mutational signatures, we analyzed WES data using an established algorithm that quantifies the enrichment of C-to-T or C-to-G mutations occurring in the TW context relative to all other cytosine mutations (9) (Fig. 6A). Similar to previous reports $(2,9,12)$, we found that the HER2-enriched subtype had the highest median APOBEC enrichments scores and the largest proportion of tumors with enrichment scores $>2$ (Fig. 6A). To estimate immune cell infiltration, we analyzed RNA-seq data for the expression of individual immune checkpoint genes or immune cell gene signatures $(56,57)$ (Supplementary Table 1). In this manner, we were able to generate quantitative estimates of APOBEC mutagenesis and immune cell infiltration within individual tumors (Supplementary Table 2). We first examined the relationship between APOBEC mutagenesis and the expression of immune signatures in HER2-enriched and basal-like breast cancers as determined by the PAM50 subtype. The basallike category includes most of the TNBCs and is considered the most immunologically active subtype of breast cancer $(58,59)$. We segregated basal-like and HER2-enriched tumors into APOBEC-high (Fig. 6B) or APOBEC-low groups (Supplementary Fig. S9A) using an APOBEC enrichment score cutoff of 2 (60). Hierarchical clustering of tumors based on immune cell signatures revealed two main clusters in each subtype. Tumors in cluster 1 had high expression of immune signatures that were reflective of an antitumor adaptive immune response, including type$1 \mathrm{~T}$ helper cells (Th1 cells), activated DCs (aDCs), $\mathrm{CD}^{+} \mathrm{T}$ cells, cytotoxic cells, interferon 
signaling pathway (IFN), major histocompatibility complex class II antigen presentation pathway (MHC-II), and checkpoint genes such as LAG3, PD1, PDL1, PDL2, CTLA4, and TIM3. Tumors

371 in cluster 2 had low expression of antitumor immune response signatures and high expression of

372 several immunosuppressive gene signatures, such as macrophages and neutrophils.

374 antitumor adaptive immune response (Fig. 6B). These results are consistent with the well-defined 375 hot TME of TNBC and their response to immune checkpoint inhibition. Surprisingly, in contrast 376 to basal-like tumors, half of the APOBEC-high HER2-enriched tumors fell within cluster 1 and 377 half within cluster 2 (Fig. 6B).

To further explore the differences in immune cell gene expression signatures between 379 basal-like, HER2-enriched, and luminal A/B tumors, we analyzed the correlation between the APOBEC signature enrichment score and each immune cell gene signature, as measured by a 381 quantitative score (Supplementary Table 3). This analysis showed that the correlation between 382 APOBEC signatures and immune cell infiltration varied by breast cancer subtype. In the basal-like 383 subtype, APOBEC signature enrichment score was positively correlated with numerous adaptive immune response gene signatures (e.g. MHC-II, aDCs, IFN, Th1 cells) and checkpoint genes (e.g. PDL1, TIM3, CTLA4), and negatively correlated with known immunosuppressive cell types 386 (macrophages, neutrophils) (Fig. 6C). Luminal A and B subtypes showed similar patterns of 387 correlation between APOBEC enrichment and immune signatures (Fig. 6C). In contrast, all but 388 one of the immune signatures (Tcm, T central memory cells), did not significantly correlate with APOBEC enrichment score in the HER2-enriched subtype, despite this subtype possessing the 
391 signature is associated with antitumor adaptive immunity gene expression in basal-like breast

392 cancer patients, but there was no evidence of association in HER2-enriched patients.

394 APOBEC-high HER2-enriched tumors in cluster 2 have increased subclonal mutations compared to tumors in cluster 1

To understand the differences in immune infiltration between APOBEC-high HER2enriched tumors in cluster 1 and 2, we first examined clinical features of tumors from each cluster. There were no statistically significant differences in estrogen receptor status, p53 status by IHC, node positivity, risk of recurrence, or pathological stage between cluster 1 and 2 of APOBEC-high HER2 tumors (data not shown). Next, in light of previous findings that tumors with more subclonal 401 mutations have a less productive immune response (52-55), we postulated that genetic 402 heterogeneity may underly the TME differences between APOBEC-high basal-like and HER2403 enriched tumors. To test this, we used the clonal phylogenies of TCGA breast cancers generated 404 by Raynaud and colleagues (61) to explore the relationship between subclonal mutations and 405 immunogenicity in human breast cancer. The HER2-enriched subtype is characterized as the breast 406 cancer subtype with the highest levels of intratumor heterogeneity, as measured by number of 407 clones in the tumor phylogeny (61). Further, HER2 ${ }^{+}$breast cancers have increased allelic 408 imbalance and chromosomal instability compared to HER2-negative tumors (62). 
414 tumors between breast cancer subtypes may be due to the levels of intratumor genetic diversity.

415 Basal-like tumors are less heterogenous and have lower APOBEC enrichment scores on average

416 than HER2-enriched tumors. Conversely, HER2-enriched tumors with high APOBEC enrichment

417 scores and high levels of genetical heterogeneity may undergo immune escape and acquire a cold

418 TME.

\section{DISCUSSION}

APOBEC mutational signatures have been identified in more than 22 different cancer types

$422(9,10)$, but the functional consequences of APOBEC activity on the tumor immune

423 microenvironment have not been explored. Here we show that APOBEC activity promotes an

424 immunologically hot, infiltrated-inflamed tumor microenvironment, leading to slowed tumor

425 growth. We find that the slowed growth of APOBEC tumors is due to an adaptive immune-

426 mediated mechanism that requires the activity of $\mathrm{CD}^{+} \mathrm{T}$ cells. APOBEC tumors exhibit a $\mathrm{T}$ cell-

427 dependent upregulation of MHC-I expression on tumor cells, and this is associated with increased

428 TCR diversity within tumors. Consistent with increased immune cell infiltration, APOBEC tumors

429 are sensitive to checkpoint inhibitors. While other studies have examined how APOBEC

430 mutagenesis sensitizes tumors to checkpoint inhibitors, this is the first study to our knowledge to

431 comprehensively define the direct consequences of APOBEC activity on the tumor immune

432 microenvironment in the absence of therapy.

433 The role of $\mathrm{CD}^{+} \mathrm{T}$ cells in the APOBEC-dependent antitumor immune response is

434 intriguing and opens up the possibility for $\mathrm{CD}^{+} \mathrm{T}$ cell-directed therapies, such as CTLA-4

435 inhibitors or $\mathrm{CD}^{+} \mathrm{T}$ cell adoptive transfer, to treat APOBEC-high patients. In a recent study of 436 murine APOBEC3-mutagenized models of TNBC, the function of $\mathrm{T}$ follicular helper cells in 
437 activating $\mathrm{B}$ cells and antibody generation was found to be required for sensitivity to anti-CTLA-

438 4/anti-PD-1 checkpoint blockade (31). Our work shows that A3B activity sensitizes HER2-driven

439 mammary tumors to anti-CTLA-4/anti-PD-1 combination therapy, while anti-PD-1 monotherapy

440 alone was ineffective. Similarly, Hollern and colleagues found that single-agent anti-PD-1 was

441 inferior to the combination therapy for TNBC (31). Thus, while the majority of immunotherapy

442 trials focus on re-invigorating $\mathrm{CD}^{+}$cytotoxic $\mathrm{T}$ cells, our findings and others suggest that

443 harnessing the activity of $\mathrm{CD}^{+}$helper $\mathrm{T}$ cells may be more beneficial for breast tumors with

444 APOBEC mutational signatures.

At the same time that APOBEC-catalyzed mutations may promote immunogenicity,

447 example, extensive evidence of APOBEC mutagenesis was found in lung cancers harboring the

448 highest burden of subclonal mutations (50), and more than $45 \%$ of subclonal mutations in cancer

449 genes could be explained by APOBEC mutagenesis (51). While there is growing interest in

450 understanding how intratumor genetic diversity impacts productive immune responses, little is

451 known about the effects of APOBEC-catalyzed subclonal diversification on tumor

452 immunogenicity. When we examined the relationship between APOBEC mutagenesis and

453 immunogenicity in human breast cancers, we observed a strong correlation between APOBEC

454 enrichment scores and immune cell gene signatures in basal-like tumors, consistent with findings

455 in other tumor types $(60,64,65)$. In contrast, there was no correlation between APOBEC

456 enrichment and immune cell signatures in HER2-enriched breast cancers. In fact, half of HER2-

457 enriched tumors with high APOBEC enrichment scores (cluster 2) had low expression of adaptive

458 immune signatures. At first glance, this was a surprising result - especially in light of our finding

459 that APOBEC activity promotes immune infiltration in HER2-driven mouse mammary tumors - 
and suggested that cluster 2 tumors may have evolved immune-suppression mechanisms that limit

461 an antitumor adaptive immune response. While the details of such mechanisms remain unknown,

462 initial insight came from examining the frequency of subclonal mutations in these tumors. Among

463 APOBEC-high tumors, immune-suppressed (cluster 2) tumors had a higher number of subclonal

464 mutations than immune-infiltrated (cluster 1) tumors. These results are reminiscent of findings

465 from other groups. For instance, in lung cancer, high clonal neoantigen burden is associated with

466 neoantigen-reactive T cells and improved immunotherapy response (55). In breast cancer, tumors

467 with high levels of heterogeneity have less infiltration of antitumor immune cells, including CD8 ${ }^{+}$

468 and $\mathrm{CD}^{+} \mathrm{T}$ cells, lower expression of PD-L1, and lower expression of cytolytic enzymes,

469 granzyme A and perforin-1 (66). These results suggest that a subset of APOBEC-high HER2

470 tumors with a high frequency of subclonal mutations can evade immune activation. These results

471 mirror our findings in mouse tumors, where clonal APOBEC tumors are controlled by the immune

472 system more profoundly than polyclonal APOBEC tumors. We propose a model (Supplementary

473 Fig. S9C), where APOBEC mutagenesis leads to immune infiltration and immunotherapy benefit

474 in both mouse models and human breast tumors yet can also foster subclonal diversification to

475 promote evasion of the immune response.

476 Therefore, to exploit the immunogenic nature of APOBEC mutations without allowing

477 acceleration of the aggressiveness of the tumor, immunotherapy could be used early on to target

478 the subclones already harboring APOBEC-catalyzed neoantigens and prevent further

479 diversification. In fact, clinical trials of immunotherapy in breast cancer show that tumors respond

480 better when administered in earlier lines of therapy (reviewed by (20)). Given our findings, prior

481 evidence in murine models $(31,32)$, and human genomic studies implicating APOBEC

482 mutagenesis in immune infiltration $(60,64,65)$ and immunotherapy response $(27-30,67)$, 
483 endogenous APOBEC mutagenesis may render human tumors responsive to immunotherapy.

484 Thus, APOBEC mutational signatures and mutational clonality may be useful biomarkers

485 predicting response to immunotherapy in women with breast cancer. This is particularly notable

486 for HER2+ breast cancer, because while the majority of reports of durable clinical benefit and

487 newly initiated immunotherapy trials are for TNBC (reviewed by (20)), HER2+ breast cancers have

488 the highest median levels of APOBEC enrichment compared to other breast cancer subtypes.

490 an antitumor immune-mediated response are in contrast to a recent study of another mutational

491 process, showing that UVB-derived mutational heterogeneity reduces antitumor immunity and

492 generates highly aggressive tumors that grow faster than non-mutagenized tumors (54).

493 Interestingly, the UVB mutational signature does not predict response to checkpoint blockade in

494 melanoma patients (29). This raises the possibility that APOBEC-mediated mutagenesis is a

495 particularly immunogenic mutational process, compared to other mutagens, such as UVB

496 irradiation, or a general increase in the TMB. For instance, APOBEC SBS signature 13, but not

497 overall TMB, correlates with immune response-specific gene expression in breast cancer (64).

498 Additionally, the APOBEC mutational signature is a better predictor of durable clinical benefit to

499 immunotherapy than total TMB in NSCLC (28). Lastly, in a cohort of patients with diverse cancer

500 types, APOBEC signatures correlate with improved immunotherapy response, independent of

501 TMB (27). It is possible that APOBEC-mediated mutations generate neoantigens that are

502 particularly immunogenic (e.g. with increased hydrophobicity (27)) or are more likely to occur in

503 highly expressed genes or regions of open chromatin (e.g. R-loops $(68,69)$ ), although human data

504 shows an inverse correlation between C-to-T mutations and gene expression (1). Future work 
bioRxiv preprint doi: https://doi.org/10.1101/2021.02.13.431068; this version posted February 14,2021 . The copyright holder for this preprint

(which was not certified by peer review) is the author/funder, who has granted bioRxiv a license to display the preprint in perpetuity. It is made available under aCC-BY-NC-ND 4.0 International license.

505 should focus on the mechanism by which the APOBEC mutational process generates 506 immunogenic neoantigens. 
MATERIALS AND METHODS

508

509

510

511

512

513

514

515

516

517

518

519

520

521

522

523

524

525

526

527

528 529

\section{Tissue culture and reagents}

All cell lines were grown at $37^{\circ} \mathrm{C}$ in $5 \% \mathrm{CO}_{2}$. SMF cells were provided by Dr. Lewis Chodosh (University of Pennsylvania) and were cultured in Dulbecco's Modified Eagle Medium (DMEM), $10 \%$ fetal bovine serum (FBS), $1 \%$ L-glutamine (Gibco 25030-081), $1 \%$ penicillin/streptomycin (Gibco 15140-122), and $5 \mu \mathrm{g} / \mathrm{mL}$ insulin (Gemini Bioproducts 700-112P). EMT6 cells were provided by the Duke Cell Culture Facility and were cultured in Waymouth's Medium 752/1, 15\% FBS, 1\% L-glutamine, and 1\% penicillin/streptomycin. BT474 cells were cultured in RPMI-1640, 10\% FBS, 1\% L-glutamine, and 1\% penicillin/streptomycin. SKBR3 cells were cultured in DMEM, 10\% FBS, 1\% L-glutamine, and 1\% penicillin/streptomycin. SMF-A3B cells were selected in $1 \mu \mathrm{g} / \mathrm{mL}$ puromycin (Sigma P8833-10MG) and $1 \mathrm{mg} / \mathrm{mL}$ neomycin (G418, Sigma, 345810-1GM). EMT6-A3B cells were selected in $4 \mu \mathrm{g} / \mathrm{mL}$ puromycin and $1 \mathrm{mg} / \mathrm{mL}$ neomycin. Doxycycline (RPI D43020-100.0) was added to the cell medium to induce the expression of A3B where specified at concentrations described. Cells were harvested for qRTPCR, deaminase activity assay, or Western blot analysis.

Cell viability assays were performed using CellTiter-Glo (Promega) according to manufacturer instructions. Cells were plated at 2,000 cells per well in an opaque 96-well plate and treated with doxycycline on day 0. Doxycycline in cell medium was refreshed every 3 days.

Colony formation assays were performed by plating cells at 2,000 cells per 10 -cm plate and cultured with doxycycline for 14 days. Doxycycline in cell medium was refreshed every 3 days. PBS was used to wash plates and $0.5 \%$ crystal violet in $25 \%$ methanal was used to stain cell colonies for 5 mins. The plates were dried overnight and imaged. Colonies were quantified using ImageJ Fiji. 
531 coverslips with $0.1 \%$ gelatin in a 24 -well plate. $1 \mu \mathrm{g} / \mathrm{mL}$ doxycycline was added, and cells were

532 cultured for 3 days prior to fixation in $4 \%$ paraformaldehyde. Coverslips were washed in PBS,

533 permeabilized in $0.5 \%$ Triton-X 100, washed in PBS, and blocked in 3\% BSA and 10\% normal

534 goat serum for 1 hour at room temperature. Coverslips were incubated with 1:800 HA-tag rabbit

535 (Cell Signaling 3724S) primary antibody overnight at $4^{\circ} \mathrm{C}$, washed, and incubated in with 1:500

536 goat anti-rabbit AF488 (Life Technologies A1103) secondary antibody for 1 hour at room

537 temperature. Coverslips were then washed in PBS, stained with DAPI for 10 minutes, and mounted

538 on slides with Prolong Gold (Thermo P36930). Slides were imaged Zeiss Axio Imager Widefield

539 fluorescence microscope.

\section{$540 \quad$ Plasmids and viral transduction}

To generate dox-inducible A3B expression in murine cancer cell lines, a 2-vector system

542 was utilized. pLVX-Tet-On Advanced plasmid containing the rtTA cassette was provided by Dr.

543 Ann Marie Pendergast (Duke University). pLenti-Tet-On-A3B plasmid containing tetracycline

544 responsive human APOBEC3B gene (NM_004900.4) that is HA-tagged on the C-terminus was

545 generated by VectorBuilder. The APOBEC3B gene contains an in-frame 66 bp SV40 T-antigen

546 intron sequence to disrupt transcription of the gene in E. coli for successful cloning without

547 introducing A3B-catalyzed mutations in the construct sequence. To generate the catalytically

548 inactive mutant of A3B (E255Q), site-directed mutagenesis of the pLenti-Tet-On-A3B plasmid

549 was performed by Genewiz. HEK293T cells were transfected with psPAX2 and pMDG.2

550 packaging plasmids (gifts from Didier Trono, EPFL, Lausanne, Switzerland; Addgene plasmids

55112559 and 12660), the lentiviral expression plasmid, PLUS reagent (Thermo 11514015), and

552 Lipofectamine 2000 (Thermo 11668019). $0.8 \mathrm{mM}$ sodium butyrate was added to cell medium 1- 
553 and 2-days post-transfection to prevent epigenetic silencing of the lentiviral vector. Lentivirus was

554 collected in the supernatant and filtered prior to concentrating with Lenti-X ${ }^{\mathrm{TM}}$ Concentrator

555 (Clontech 631231) manufacturer protocol.

556 To generate SMF-A3B and EMT6-A3B cell lines, SMF cells and EMT6 cells were

557 transduced at $50 \%$ confluency in 6 -well plates with $1 \mathrm{~mL}$ of concentrated lentivirus and $6 \mu \mathrm{g} / \mathrm{mL}$

558 polybrene (Sigma 107689) at $1000 x g$ and $33^{\circ} \mathrm{C}$ for 2 hours. Cells transduced with pLVX-Tet-On

559 Advanced lentivirus were selected in neomycin for at least 10 days. Cells were then transduced

560 with pLenti-Tet-On-A3B lentivirus and selected in puromycin for an additional 14 days.

561 Animal work

Animal care and animal experiments were performed with the approval of, and in

563 accordance with, guidelines of the Duke University IACUC. Mice were housed under barrier

564 conditions with 12-hour light/12-hour dark cycles. Female FVB mice (FVB/NJ; used with SMF

565 cells) and female BALB/c mice (BALBc/J; used with EMT6 cells) were obtained from The

566 Jackson Laboratory. Female outbred athymic nude mice (J:NU) and female NOD.Cg-Prkdc scid

$567 I l 2 \mathrm{rg}^{t m 1 W_{j l} / \mathrm{SzJ}}$ (NSG) mice were obtained from The Jackson Laboratory.

Tumor cell lines were implanted in bilateral $4^{\text {th }}$ inguinal mammary fat pads of 6-8 week old

569 female recipient mice. $2 \times 10^{6}$ SMF-A3B cells or $2 \times 10^{4}$ EMT6-A3B cells in complete cell medium

570 were used for implantation. Tumors were monitored for growth, measured using calipers 2-3 times

571 per week, and sacrificed at experimental endpoint or when tumors reached 10-15 mm in diameter.

572 Tumor volume was calculated using $\left(\mathrm{L}^{*} \mathrm{~W} * \mathrm{~W} * \pi\right) / 6$, where $\mathrm{L}$ is length of the longer side and $\mathrm{W}$ is

573 length of the shorter side. Where indicated, $1 \mathrm{mg} / \mathrm{mL}$ of doxycycline supplemented with $5 \%$

574 sucrose was added to mouse drinking water 2 days prior to tumor cell implantation. 
5761 prior to implantation, then continued twice weekly until endpoint. $300 \mu \mathrm{g}$ of anti-CD8 (BioXCell

$577 \mathrm{BE} 0117$ ), or $300 \mu \mathrm{g}$ of anti-IgG2b isotype control (BioXCell BE0090), was used for CD8

578 depletion alone. $200 \mu \mathrm{g}$ of anti-CD8 (BioXCell BE0117) and $200 \mu \mathrm{g}$ of anti-CD4 (BioXCell

579 BE0003-1), or $400 \mu \mathrm{g}$ of anti-IgG2b isotype control (BioXCell BE0090), was used for CD8/CD4

580 dual depletion. For anti-PD-1 monotherapy, antibodies were administered when the majority of

581 tumors reached $5 \mathrm{~mm}$ in diameter, for a total of 3 doses in one week (day 13, 15, 17) and 3 doses

582 in the next week (day 20, 22, 24). $200 \mu \mathrm{g}$ of anti-PD-1 (BioXCell BE0146), or $300 \mu \mathrm{g}$ of anti-

583 IgG2b (BioXCell BE0090), was used for monotherapy. For combination anti-PD-1/anti-CTLA-4

584 therapy, antibodies were administered when the majority of tumors reached $5 \mathrm{~mm}$ in diameter and

585 continued twice weekly until endpoint. $200 \mu \mathrm{g}$ of anti-PD-1 (BioXCell BE0146) and $200 \mu \mathrm{g}$ of

586 anti-CTLA-4 (BioXCell BE0164), or $400 \mu \mathrm{g}$ of anti-IgG2b isotype control (BioXCell BE0090),

587 was used.

\section{Flow cytometry}

Bilateral tumors were harvested and aggreged for each mouse, then minced into small

590 chunks. Tumor chunks were digested with warmed digestion buffer containing $300 \mathrm{U} / \mathrm{mL}$

591 collagenase (StemCell 554656) and $100 \mathrm{U} / \mathrm{mL}$ hyaluronidase (StemCell 554656) at $37{ }^{\circ} \mathrm{C}$ for 1

592 hour, vortexing every 15 minutes. Digested tumors were incubated in ACK lysis buffer for 5

593 minutes to lysis red blood cells. Tumors were centrifuged, washed in stain buffer (BD Biosciences

594 554656), decanted, and resuspended in Dispase II (5 mg/mL; StemCell 7913) and DNase I (100

$595 \mu \mathrm{g} / \mathrm{mL}$; Worthington Biochemical LS002006) for 5 minutes, mixing. Tumors were then passed

596 through $70 \mu \mathrm{m}$ strainer, washed in stain buffer, counted, and $1 \times 10^{6}$ cells in $100 \mu \mathrm{L}$ of stain buffer

597 were added to 96-well untreated v-bottom plate for staining. Prior to intracellular antigen staining, 
cells were activated using $2 \mu \mathrm{L}$ of leukocyte activation cocktail with GolgiPlug (BD Biosciences 550583 ) for 3 hours at $37^{\circ} \mathrm{C}$ and $5 \% \mathrm{CO}_{2}$. cells in PBS according to manufacturer protocol for 30 minutes at $4^{\circ} \mathrm{C}$ in the dark. Cells were washed in PBS three times and resuspended in $100 \mu \mathrm{L}$ of PBS for antibody surface staining. $2 \mu \mathrm{L}$ of CD16/CD32 Fc Block antibody (BD Biosciences 553141) was added for 10 minutes at $4^{\circ} \mathrm{C}$ in the dark. Surface antigen antibodies were added at dilutions listed below and incubated for 30 minutes at $4{ }^{\circ} \mathrm{C}$ in the dark. Cells were washed in PBS and transferred to falcon tubes for analysis. Biosciences 560409) or BD Cytofix ${ }^{\mathrm{TM}}$ Fixation Buffer (BD Biosciences 554655) for 30 minutes at $4^{\circ} \mathrm{C}$ in the dark. Cells were washed and stored at $4^{\circ} \mathrm{C}$ in the dark overnight. Cells were permeabilizated in either Foxp3 permeabilization buffer (BD Biosciences 560409) for 30 minutes at $37^{\circ} \mathrm{C}$ or BD perm/wash buffer for 15 minutes at $4^{\circ} \mathrm{C}$. Cells were washed in PBS and resuspended in $100 \mu \mathrm{L}$ of PBS for intracellular antigen staining using the antibody dilutions listed below and

612 incubated for 25 minutes at room temperature in the dark. Cells were washed in PBS and 613 transferred to falcon tubes for analysis. analyzed using FlowJo software (TreeStar, Ashland, OR). Fluorescence minus one (FMO; all

616 antibodies in the panel, except for one) was used to determine proper gating of individual cell

617 types. Individual cell type compartments were represented as either the percentage of total CD45

618 cells or the percentage of total live cells. Treg and Th2 cell compartments were represented as 619 percentage of total $\mathrm{CD}^{+} \mathrm{T}$ cells. The correlation between immune cell frequency and tumor volume was calculated using the mean volume of bilateral tumors at endpoint. 


\begin{tabular}{|c|c|c|c|c|c|c|}
\hline $\begin{array}{l}\text { Marker/Cell } \\
\text { Type }\end{array}$ & Antigen & Fluorophore & Clone & Vendor & $\begin{array}{l}\text { Catalog } \\
\text { Number }\end{array}$ & Dilution \\
\hline PD-L1 & $\begin{array}{l}\text { PD-L1 } \\
\text { (CD274) }\end{array}$ & BV421 & MIH5 & $\mathrm{BD}$ & 564716 & $1: 100$ \\
\hline PD-1 & $\begin{array}{l}\text { PD-1 } \\
\text { (CD279) }\end{array}$ & AF647 & RMP1-30 & $\mathrm{BD}$ & 566715 & $1: 20$ \\
\hline Dendritic Cell & CD11c & PECy7 & HL3 & $\mathrm{BD}$ & 558079 & $1: 100$ \\
\hline Dendritic Cell & $\begin{array}{l}\text { MHC-II I-A } \\
\text { I-E }\end{array}$ & AF488 & M5/114.15.2 & $\mathrm{BD}$ & 562352 & $1: 100$ \\
\hline Dendritic Cell & CD103 & $\mathrm{PE}$ & M290 & $\mathrm{BD}$ & 561043 & $1: 100$ \\
\hline Epithelial Cell & $\begin{array}{l}\text { EpCAM } \\
\text { (CD326) }\end{array}$ & FITC & G8.8 & Biolegend & 118207 & $1: 500$ \\
\hline Leukocyte & CD45 & PECy5 & 30-F11 & $\mathrm{BD}$ & 561870 & $1: 200$ \\
\hline Leukocyte & CD45 & PECy7 & 30-F11 & $\mathrm{BD}$ & 552848 & $1: 200$ \\
\hline Leukocyte & CD45 & APC & 30-F11 & BD & 561870 & $1: 200$ \\
\hline Leukocyte & CD45 & V450 & 30-F11 & $\mathrm{BD}$ & 560501 & $1: 200$ \\
\hline Leukocyte & CD45 & $\mathrm{PE}$ & 30-F11 & $\mathrm{BD}$ & 561087 & $1: 500$ \\
\hline Leukocyte & CD45 & PerCP-Cy5.5 & 30-F11 & $\mathrm{BD}$ & 550994 & $1: 200$ \\
\hline Macrophage & $\mathrm{F} 4 / 80$ & APC & BM8 & BioLegend & 123116 & $1: 50$ \\
\hline Macrophage & $\mathrm{F} 4 / 80$ & AF647 & T45-2342 & $\mathrm{BD}$ & 565853 & $1: 50$ \\
\hline Monocyte & CD11b & $\mathrm{PE}$ & $\mathrm{M} 1 / 70$ & BD & 561689 & $1: 50$ \\
\hline Monocyte & CD11b & PECy7 & $\mathrm{M} 1 / 70$ & $\mathrm{BD}$ & 561098 & $1: 100$ \\
\hline Monocyte & CD11b & APCCy7 & $\mathrm{M} 1 / 70$ & $\mathrm{BD}$ & 557657 & $1: 100$ \\
\hline NK Cell & NK1.1 & APC & PK136 & $\mathrm{BD}$ & 561117 & $1: 100$ \\
\hline NK Cell & CD49b & APC & DX5 & BioLegend & 108909 & $1: 50$ \\
\hline T Cell & CD3e & PECy7 & $145-2 \mathrm{C} 11$ & $\mathrm{BD}$ & 561100 & $1: 100$ \\
\hline T Cell & CD3e & $\mathrm{PE}$ & $145-2 \mathrm{C} 11$ & BD & 561824 & $1: 100$ \\
\hline T Cell & CD3e & PerCP-Сy5.5 & $145-2 \mathrm{C} 11$ & $\mathrm{BD}$ & 561108 & $1: 100$ \\
\hline T Cell & CD4 & APCCy7 & GK1.5 & BD & 561830 & $1: 100$ \\
\hline T Cell & CD8a & APC & $53-6.7$ & BD & 561093 & $1: 200$ \\
\hline T Cell & CD8a & AF488 & $53-6.7$ & $\mathrm{BD}$ & 557668 & $1: 100$ \\
\hline T Cell & Foxp3 & AF488 & MF23 & $\mathrm{BD}$ & 560407 & $1: 200$ \\
\hline T Cell & Tbet & BV421 & O4-46 & $\mathrm{BD}$ & 563318 & $1: 100$ \\
\hline T Cell & Gata3 & AF647 & L50-823 & $\mathrm{BD}$ & 560068 & $1: 200$ \\
\hline T Cell & IL-4 & APC & 11B11 & Biolegend & 504105 & $1: 100$ \\
\hline Granzyme B & Granzyme B & FITC & GB11 & BD & 515403 & $1: 50$ \\
\hline Interferon-y & IFNy & BV421 & XMG1.2 & $\mathrm{BD}$ & 563376 & $1: 40$ \\
\hline Fc Block & CD16/CD32 & - & $2.4 \mathrm{G} 2$ & $\mathrm{BD}$ & 553141 & $1: 50$ \\
\hline
\end{tabular}

621

\section{2 qRT-PCR and Western blotting}

623

RNA was extracted, cDNA generated, and gene expression level determined by qRT-PCR

624 as previously described in (70). Taqman Probes (Thermo 4331182): APOBEC3B, 

Hs00358981_m1; ACTB, Hs01060665_g1; Actb, Mm02619580_g1; Foxp3, Mm00475162_m1;

626 Gzma, Mm01304452_m1; Tbx21, Mm00450960_m1; Prf1, Mm00812512_m1. mRNA

627 expression was normalized to $\beta$-actin and presented as the relative fold change. To compare A3B

628 expression levels between murine cell lines (SMF-A3B and EMT6-A3B) and human cell lines

629 (BT474), A3B expression was not normalized to account for differences in $\beta$-actin expression

630 between mouse and human cells; fold change of relative $\mathrm{Ct}$ value was presented.

For Western blotting, cells were treated doxycycline as described and harvested. Cells were

632 lysed in RIPA buffer and 1x Halt Proteinase/Phosphatase Inhibitor (Invitrogen 78444). Protein

633 concentration in the supernatant was determined by Bradford assay. Laemmli Sample Buffer

634 (BioRad 1610747) was added to diluted protein samples and denatured at $95^{\circ} \mathrm{C}$ for 5 minutes. 20

$635 \mu \mathrm{g}$ of denatured protein was loaded into wells of 10-15\% SDS-PAGE gel and ran at 90-125 V for

6361 hour. Gel was transferred to immunoblot membrane using wet transfer at $90 \mathrm{~V}$ for 1 hour.

637 Membranes were incubated with blocking buffer for 1 hour at room temperature and then primary

638 antibodies at dilutions listed below overnight at $4^{\circ} \mathrm{C}$. Membranes were washed in PBS-Tween 20

639 and incubated with secondary antibodies at dilutions listed below for 1 hour at room temperature

640 in the dark. Membranes were then washed and imaged using a Li-Cor Odyssey infrared imaging

641 system and analyzed in ImageStudio Lite software (Li-Cor Biosciences).

\begin{tabular}{|l|l|l|l|}
\hline Antibody & Vendor & Catalog Number & Dilution \\
\hline HA-tag Rabbit & Cell Signaling & $3724 \mathrm{~S}$ & $1: 1000$ \\
\hline$\alpha$-Tubulin Mouse & Cell Signaling & 3873 & $1: 2000$ \\
\hline$\gamma H 2 A X($ Ser139) Rabbit & Cell Signaling & $2577 \mathrm{~S}$ & $1: 1000$ \\
\hline H2A Mouse & Cell Signaling & $3636 \mathrm{~S}$ & $1: 1000$ \\
\hline Cleaved PARP (Asp214) Mouse & Cell Signaling & $9544 \mathrm{~S}$ & $1: 1000$ \\
\hline
\end{tabular}




\begin{tabular}{|l|l|l|l|}
\hline Goat anti-Mouse IRDye800 & Li-Cor & $926-32210$ & $1: 5000$ \\
\hline Goat anti-Rabbit AF680 & Thermo & A-21076 & $1: 5000$ \\
\hline
\end{tabular}

643 Tissues, immunohistochemistry, and immunofluorescence

644 Tumors were harvested and fixed in $10 \%$ normal formalin overnight before paraffin-

645 embedding for immunohistochemistry by Duke Pathology Research Immunohistology Lab (Duke

646 University, Durham, NC). Slides were imaged at 4 fields of view per tumor with Zeiss Axio Imager

647 Widefield fluorescence microscope.

Tumors were harvested and frozen in OCT for immunofluorescence staining. Slides were

649 fixed in $4 \%$ paraformaldehyde for 10 minutes. Slides were washed in PBS, permeabilized in $0.5 \%$

650 Triton-X 100 for 20 minutes, washed in PBS, and blocked in 3\% BSA and 10\% normal goat serum

651 for 1 hour at room temperature. Slides were incubated with primary antibodies listed below

652 overnight at $4^{\circ} \mathrm{C}$, washed, and incubated in with secondary antibodies listed below for 1 hour at

653 room temperature. Slides were then washed in PBS, stained with DAPI for 10 minutes, and

654 coverslips were mounted on slides with Prolong Gold (Thermo P36930). For $\gamma \mathrm{H} 2 \mathrm{AX}$ foci

655 quantification, 8 fields of view were imaged per slide with Leica SP5 Inverted Confocal

656 fluorescence microscope. For assessing expression of HA-tagged A3B in tumors, slides were

657 imaged with Zeiss Axio Imager Widefield fluorescence microscope. Images were analyzed with

658 ImageJ Fiji.

\begin{tabular}{|l|l|l|l|}
\hline Antibody & Vendor & Catalog Number & Dilution \\
\hline CD3 Rabbit & Thermo & RM-9107-S & $1: 100(\mathrm{IHC})$ \\
\hline CD45 Rat & BD Biosciences & 550939 & $1: 50(\mathrm{IHC})$ \\
\hline HA-tag Rabbit & Cell Signaling & $3724 \mathrm{~S}$ & $1: 800(\mathrm{IF})$ \\
\hline
\end{tabular}




\begin{tabular}{|l|l|l|l|}
\hline$\gamma$ H2AX (Ser139) Rabbit & Cell Signaling & 2577S & $1: 800$ (IF) \\
\hline Goat anti-Rabbit AF488 & Life Technologies & A1103 & $1: 500$ (IF) \\
\hline
\end{tabular}

\section{Cytidine deaminase activity assay} inhibitor (Sigma P8340). Protein concentration of the supernatant was determined using DC ${ }^{\mathrm{TM}}$ Protein Assay (BioRad) and manufacturer protocol. $10 \mu \mathrm{g}$ of protein was incubated for 2 hours at $37^{\circ} \mathrm{C}$ with $4 \mathrm{pmol}$ of oligonucleotide listed below, $0.5 \mu \mathrm{L}$ of uracil DNA glycosylase enzyme (NEB

673 for 1 hour at $150 \mathrm{~V} .5 \mu \mathrm{L}$ of denatured sample was added per well and ran at $150 \mathrm{~V}$ for $30-45$

674 minutes. Gels were imaged with Li-Cor Odyssey infrared imaging system and analyzed in

675 ImageStudio Lite software (Li-Cor Biosciences) to quantify the percent of deamination.

676 Oligonucleotide containing cytosine:

678 Positive control oligonucleotide containing uracil: 
RNA-sequencing and analysis

681

RNA was isolated from tumors using the RNeasy kit (Qiagen). RNA was sequenced using

682 Stranded mRNA-seq libraries and the NovaSeq 6000 S1 sequencing platform with 50 bp paired-

683 end reads by the Duke GCB Sequencing and Genomic Technologies Shared Resource (Duke

684 University, Durham, NC).

685

RNA-seq data was trimmed with Trim Galore! (Galaxy Version 0.6.3; Krueger, F.,

Babraham Institute, http://www.bioinformatics.babraham.ac.uk/projects/trim_galore/) and then

687 FastQC (Galaxy Version 0.72+galaxy1; Andrews, S. (n.d.). FastQC A Quality Control tool for

Throughput

Sequence

Data.

Retrieved

from

http://www.bioinformatics.babraham.ac.uk/projects/fastqc/) was used to assess quality. Reads

690

were aligned to the GRCm38 reference mouse genome using RNA STAR (Galaxy Version

2.7.5b)(71) and vM25 annotation file downloaded from the Gencode server. Reads were counted

with featureCounts (Galaxy Version 1.6.4+galaxy2)(72) and differential gene expression analysis

was performed with DESeq2 (Galaxy Version 2.11.40.6+galaxy1)(73).

Gene ontology (GO) analysis was performed using GO Ontology database (DOI:

10.5281/zenodo.4033054 Released 2020-09-10)(74,75) for the $\log 2$ fold change of genes with a

696 FDR adjusted p-value < 0.05. Gene set enrichment analysis (GSEA) was performed using GSEA

697 v4.1.0(76,77) using preranked gene list and Hallmark v7.2 gene set database at 1,000 permutations.

698 In silico flow cytometry was used to compute immune cell fractions with CIBERSORTx(78) and

699 the LM22 dataset.

\section{T cell receptor-sequencing and analysis}

701

RNA was isolated from tumors using the RNeasy kit (Qiagen) and TCR beta chain libraries 
to a final pool concentration of $4 \mathrm{nM}$ and diluted to a final concentration of $13.5 \mathrm{pM}$, including a

704 5-10\% PhiX Control v3 spike-in. Libraries were sequencing using MiSeq600 v3 300 bp paired-

705 end reads. MiXCR(79) was used to calculate clonotype frequencies with recommended settings

706 and vegan R package (Jari Oksanen, F. Guillaume Blanchet, Michael Friendly, Roeland Kindt,

707 Pierre Legendre, Dan McGlinn, Peter R. Minchin, R. B. O'Hara, Gavin L. Simpson, Peter Solymos,

708 M. Henry H. Stevens, Eduard Szoecs and Helene Wagner (2020). vegan: Community Ecology

709 Package. R package version 2.5-7. https://CRAN.R-project.org/package=vegan) was used to

710 calculate Shannon entropy diversity index. $\mathrm{DE}_{50}$ was calculated as the number of clonotypes

711 occupying the top $50 \%$ of read counts, divided by the total number of read counts.

\section{ELISpot}

713

Spleens and tumors were harvested from 4 APOBEC tumor-bearing mice. Single cell

714 splenocytes suspensions were generated and cryopreserved. Tumor chunks were snap-frozen in

715 liquid nitrogen. For co-culture, splenocytes were thawed at $37^{\circ} \mathrm{C}$, washed 3 times in splenocyte

716 medium (RPMI, 10\% FBS, and 1\% penicillin/streptomycin), and counted. Tumor chunks were

717 thawed on ice, lysed using 4 rounds of $-80^{\circ} \mathrm{C}$ freeze/thaw cycles, and protein concentrations were

718 determined from the supernatant using Bradford assay. $1 \times 10^{6}$ splenocytes from APOBEC tumor-

719 bearing mice were co-cultured for 48 hours with $100 \mu \mathrm{g} / \mathrm{mL}$ of tumor lysate protein. $2.5 \times 10^{5}$

720 splenocytes from naïve mice were co-cultured for 48 hours with $1 \mu \mathrm{g} / \mathrm{mL}$ of concanavalin A as a

721 positive control or alone as a negative control. Mouse IFN- $\gamma$ ELISpot PLUS kit (MABTECH

722 3321-4APT-2) manufacturer protocol was followed for development of the spots. Plates were dried

723 overnight and imaged and quantified using CTL ImmunoSpot 7.0.26.0 software.

\section{Bioinformatics analysis of human breast cancers}


To estimate immune cell infiltration, we quantified the gene expression of individual

726 immune checkpoint genes or immune cell gene signatures $(56,57)$ collapsed into one value for

727 each signature using a PCA-based method (see Supplementary Table 1 for gene lists). Raw gene

728 counts from RNA-seq experiments for TCGA-BRCA patients were first queried from the National

729 Cancer Institute Genomic Data Commons (GDC) (80) using the R package TCGAbiolinks

730 (v2.12.6) (81), and normalized to effective library sizes calculated by the Trimmed Mean of M-

731 values (TMM) (82) method and transformed by the voom method (83) implemented in the R

732 packages edgeR (v3.28.0) (84) and Limma (v3.42.0) (85), respectively. For each gene signature,

733 the first principal component (PC1) of a PCA model was used to summarize the gene expression

734 values of the signature into a single score.

735 To calculate APOBEC enrichment score, single nucleotide polymorphisms (SNP) data

736 called by the somatic mutation caller MuTect2 (86) were also queried from the GDC. For each

737 tumor, an APOBEC mutagenesis enrichment score was calculated based on $\mathrm{C}>\mathrm{T}$ mutations

738 occurring in TCW motifs as described by Roberts et al (9).

739 Heatmaps of the relative expression of immune cell gene signatures in APOBEC-high and

740 APOBEC-low tumors were created using $\mathrm{R}$ package Morpheus

741 (https://software.broadinstitute.org/morpheus). Samples were grouped by subtype (HER2-

742 enriched or basal-like), and Euclidian hierarchical clustering and cutting the dendrogram was used

743 to identify 2 main immune clusters in the HER2-enriched subtype and 2 main immune clusters in

744 the basal-like subtype (immune cluster 1 and cluster 2).

745 To measure the correlation of APOBEC enrichment score and immune gene signatures in

746 samples based on subtype, Spearman's rho was calculated, and the significance was determined 
747 by Spearman's rank correlation test. P-values were adjusted for multiple testing using the

748 Benjamini-Hochberg method to control the false discovery rate.

To assess differences in genetic heterogeneity between immune clusters, the number of

750 subclonal mutations per TCGA sample was downloaded from (61).

751 Gene expression-based classifier of APOBEC mutagenesis

APOBEC enrichment scores were calculated from TCGA tumors as in methods described

753 above. We performed classification to nearest centroids to identify sets of genes that would

754 distinguish individuals with high APOBEC enrichment from those without (87). After constructing

755 a matrix of log-2 transformed, median-centered gene expression values for TCGA-BRCA samples,

756 we filtered genes to the top 5\% most differentially expressed ( $\mathrm{N}=1,026$ genes) between APOBEC-

757 high and APOBEC-low samples using the samr package (R. Tibshirani, Michael J. Seo, G. Chu,

758 Balasubramanian Narasimhan and Jun Li (2018). samr: SAM: Significance Analysis of

759 Microarrays. R package version 3.0. https://CRAN.R-project.org/package=samr). We performed

76010 -fold cross validation by randomly splitting the TCGA samples into 10 groups and training the

761 classifier on nine of these groups (training set), leaving the remaining group to serve as an internal

762 validation set (test set). In each of the 10 iterations of training, we varied the number of genes used

763 to predict each APOBEC group from 1 to 50 and assessed model performance by calculating

764 sensitivity and specificity in both training and test sets. Mean sensitivity compared to APOBEC

765 enrichment calls derived from whole-exome-sequencing across each of the 10 folds ranged from

$766 \quad 61-71 \%$ in training sets, with the maximum test set sensitivity reached at 5 genes per group

767 (Supplementary Fig. S10A, B). We chose the final number of genes based on the maximum

768 Youden's index (sensitivity + specificity -1$)$. The maximum Youden's index for test data was

769 achieved using 5 genes per group $(\mathrm{Y}=0.30)$, suggesting that a 10-gene classifier was optimal for 
770 prediction (Supplementary Fig. S10C). Applied to the full TCGA breast cancer cohort, the

771 predictor achieved 69\% sensitivity and 61\% specificity against APOBEC enrichment calls from

772 whole-exome sequencing data, for an overall accuracy of 63\% (Supplementary Fig. S10D). This

773 accuracy is consistent with what would be expected given the observed instability in signature

774 detection when resampling mutations within an individual, particularly in contexts of low mutation

775 frequency $(41,42)$. Finally, the expression-based predictor was applied to RNA-seq data from a

776 sample of 12 mouse tumors from NSG mice (6 A3B-expressing tumors and 6 control tumors) to

777 classify tumors demonstrating the APOBEC mutational signature. In this instance, sensitivity and

778 specificity were calculated using A3B/control status as the gold standard.

779 Statistical reporting

780 One-way ANOVA and Tukey's multiple comparisons test was used to assess statistical

781 significance of qRT-PCR gene expression, colony formation assay, and MHC-I expression by flow

782 cytometry. One-way ANOVA and Sidak's multiple comparisons test were used to assess statistical

783 significance of the mouse tumor volume on a single day as indicated, differences in APOBEC

784 enrichment score from human data, and the number of subclonal mutations in immune clusters

785 from human data. Two-way ANOVA and Dunnett's multiple comparisons test was used to

786 determine the statistical significance of differential cell growth in vitro using CellTiter Glo assay.

787 Two-way repeated-measures ANOVA and Tukey's multiple comparison was used to measure

788 statistical significance of changes in tumor volume over time in vivo. The adjusted p-values are

789 reported for each.

Fisher's exact test was used to assess differences in response to checkpoint inhibition

791 (CR/PR by percent change in tumor volume from treatment start day). Student's t-test was used to

792 test the statistical significance of differences in tumor mass at endpoint, flow cytometry, IHC/IF, 
and TCR-seq diversity measurements. Student's t-test p-values are reported. Statistical analysis was performed and graphs were created in R version 4.0.2 or using GraphPad Prism version 8.0.1.

Source code to reproduce analyses of gene expressed-based APOBEC classifier and to

797 reproduce analyses of APOBEC enrichment and quantification of immune signature gene expression is available at https://github.com/ashleydimarco/alvarezlab-APOBEC

\section{Author contributions}

Ashley V. DiMarco: conceptualization, data curation, formal analysis, supervision,

801 validation, investigation, visualization, methodology, writing-original draft, project

802 administration; Xiaodi Qin: data curation, formal analysis, methodology, writing - review and

803 editing for APOBEC enrichment score, immune gene signatures, and correlation analysis of

804 TCGA data; Sarah Van Alsten: data curation, formal analysis, methodology, writing — review

805 and editing for gene expressed-based classifier of APOBEC mutagenesis; Brock McKinney: data

806 curation, resources; Nina Marie G. Garcia: data curation, writing - review and editing; Jeremy

807 Force: methodology; Brent A. Hanks: supervision, writing-review and editing for depletion

808 and checkpoint inhibitor studies; Melissa A. Troester: supervision, writing - review and editing

809 for gene expressed-based classifier of APOBEC mutagenesis; Kouros Owzar: supervision,

810 writing - review and editing for APOBEC enrichment score, immune gene signatures, and

811 correlation analysis of TCGA data; Jichun Xie: supervision, writing - review and editing for

812 APOBEC enrichment score, immune gene signatures, and correlation analysis of TCGA data;

813 James V. Alvarez: conceptualization, supervision, funding acquisition, project administration,

814 writing - review and editing.

\section{Acknowledgements}


We thank Dr. Lewis Chodosh (University of Pennsylvania) for providing the SMF cell line.

817 We thank Dr. Michael Plebanek and Dr. Nicolas Devito (Duke University) for technical advice

818 with flow cytometry and ELISpot. We thank Elizabeth Mendes and Alexandra Bennion (Duke

819 University) for providing technical assistance, and Dr. Andrea Walens (University of North

820 Carolina at Chapel Hill) for reviewing the manuscript. We thank the Duke Pathology Research

821 Immunohistology Lab for paraffin processing and IHC staining of tissue. We thank Dr. Nicolas

822 Devos (Duke University) and the Duke University School of Medicine Sequencing and Genomic

823 Technologies Shared Resource for providing library preparation and sequencing for RNA-seq and

824 WES analyses, and sequencing for TCR-seq analysis. This work was funded by the National

825 Cancer Institute under award R01CA208042 (to J.V.A.) and T32-CA009111 (to A.V.D.), as well

826 as the American Cancer Society under award 132556-RSG-18-130-CCG (to J.V.A.) and by startup

827 funds from the Duke Cancer Institute, the Duke University School of Medicine, the Whitehead

828 Foundation (to J.V.A.), and the National Institutes of Health under T32-GM007184 (to A.V.D.). 
SUPPLEMENTARY MATERIAL

\section{Supplementary Figures S1-S10}

831 Supplementary Table S1: Spreadsheet of gene lists for immune cell gene signatures for analyses

832 in Figure 6 and Supplementary Figure S9. The genes that were absent from the TCGA-BRCA

833 RNA-seq dataset are colored in red.

834 Supplementary Table S2: Spreadsheet containing TCGA-BRCA patient ID and data used for 835 analyses in Figure 6 and Supplementary Figure S9. Column descriptions:

836 Sample_ID - TCGA sample identifier

837 Cluster_Number - APOBEC-high or -low immune cluster number (e.g. “APOBEC-high HER2-

838 1” refers to APOBEC-high HER2 subtype Immune Cluster 1)

839 Age_Median - patient age

840 ER.Status - clinical ER status

841 PR.Status - clinical PR status

842 Her2.Status - clinical HER2 status

843 PAM50 - PAM50 subtype

844 Pathologic_stage - clinical pathologic stage

845 Histological_type - clinical histological type

846 n_C_mut - number of $\mathrm{C}>\mathrm{T} / \mathrm{G}($ or $\mathrm{G}>\mathrm{A})$ mutations

847 n_C_con - number of C (or G) within the 41-nucleotide region centered on the $\mathrm{C}>\mathrm{T} / \mathrm{G}$ (or $\mathrm{G}>\mathrm{C} / \mathrm{A}$ )

848 mutations

849 n_TCW_mut - number of $\mathrm{C}>\mathrm{T} / \mathrm{G}$ ( or $\mathrm{G}>\mathrm{C} / \mathrm{A}$ ) mutations in TCW (or WGA) motifs

850 n_TCW_con - number of TCW (or WGA) motifs within the 41-nucleotide region centered on the

851 mutated motifs, TCW to TTW/TGW (or WGA to WAA/WCA). 
852 APOBEC - APOBEC enrichment score

853 Number_of_Subclonal_Mutations - number of subclonal mutations from Raynaud et al. 2018

854 The remaining columns are principal component analysis (PCA)-collapsed $\log 2$ normalized gene

855 expression of immune cell gene signatures from RNA-seq data.

856 Supplementary Table S3: Spreadsheet of correlations between APOBEC enrichment score and

857 immune cell signatures used for analyses in Figure 6. Column descriptions:

858 PAM50 - PAM50 subtype

859 rho - Spearman's rho value from correlation analysis

860 pvalue - p-value from correlation analysis

861 adjusted_pvalue - adjusted p-value from correlation analysis 


\section{REFERENCES}

1. Nik-Zainal S, Alexandrov LB, Wedge DC, Van Loo P, Greenman CD, Raine K, et al. Mutational processes molding the genomes of 21 breast cancers. Cell. 2012;149:979-93.

2. Alexandrov LB, Nik-Zainal S, Wedge DC, Aparicio SAJR, Behjati S, Biankin AV, et al. Signatures of mutational processes in human cancer. Nature. 2013;500:415-21.

3. Alexandrov LB, Kim J, Haradhvala NJ, Huang MN, Tian Ng AW, Wu Y, et al. The repertoire of mutational signatures in human cancer. Nature. 2020;578:94-101.

4. Roberts SA, Sterling J, Thompson C, Harris S, Mav D, Shah R, et al. Clustered mutations in yeast and in human cancers can arise from damaged long single-strand DNA regions. Mol Cell. 2012;46:424-35.

5. Taylor BJ, Nik-Zainal S, Wu YL, Stebbings LA, Raine K, Campbell PJ, et al. DNA deaminases induce break-associated mutation showers with implication of APOBEC3B and 3A in breast cancer kataegis. Elife. 2013;2:e00534.

6. Supek F, Lehner B. Clustered Mutation Signatures Reveal that Error-Prone DNA Repair Targets Mutations to Active Genes. Cell. 2017;170:534-547.e23.

7. Swanton C, McGranahan N, Starrett GJ, Harris RS. APOBEC enzymes: mutagenic fuel for cancer evolution and heterogeneity. Cancer Discov. 2015;5:704-12.

8. Roberts SA, Gordenin DA. Clustered and genome-wide transient mutagenesis in human cancers: Hypermutation without permanent mutators or loss of fitness. Bioessays. 2014;36:382-93.

9. Roberts SA, Lawrence MS, Klimczak LJ, Grimm SA, Fargo D, Stojanov P, et al. An APOBEC cytidine deaminase mutagenesis pattern is widespread in human cancers. Nat Genet. 2013;45:970-6.

10. Burns MB, Temiz NA, Harris RS. Evidence for APOBEC3B mutagenesis in multiple human cancers. Nat Genet. 2013;45:977-83.

11. Nik-Zainal S, Davies H, Staaf J, Ramakrishna M, Glodzik D, Zou X, et al. Landscape of somatic mutations in 560 breast cancer whole-genome sequences. Nature. 2016;534:47-54.

12. Kanu N, Cerone MA, Goh G, Zalmas L-P, Bartkova J, Dietzen M, et al. DNA replication stress mediates APOBEC3 family mutagenesis in breast cancer. Genome Biol. 2016;17:185.

13. van Rooij N, van Buuren MM, Philips D, Velds A, Toebes M, Heemskerk B, et al. Tumor exome analysis reveals neoantigen-specific T-cell reactivity in an ipilimumab-responsive melanoma. J Clin Oncol. 2013;31:e439-42.

14. Castle JC, Kreiter S, Diekmann J, Löwer M, van de Roemer N, de Graaf J, et al. Exploiting the mutanome for tumor vaccination. Cancer Res. 2012;72:1081-91.

15. Le DT, Uram JN, Wang H, Bartlett BR, Kemberling H, Eyring AD, et al. PD-1 Blockade in Tumors with Mismatch-Repair Deficiency. N Engl J Med. 2015;372:2509-20.

16. Le DT, Durham JN, Smith KN, Wang H, Bartlett BR, Aulakh LK, et al. Mismatch repair deficiency predicts response of solid tumors to PD-1 blockade. Science. 2017;357:409-13.

17. Rizvi NA, Hellmann MD, Snyder A, Kvistborg P, Makarov V, Havel JJ, et al. Cancer immunology. Mutational landscape determines sensitivity to PD-1 blockade in non-small cell lung cancer. Science. 2015;348:124-8.

18. Vonderheide RH, Domchek SM, Clark AS. Immunotherapy for breast cancer: what are we missing? Clin Cancer Res. 2017;23:2640-6.

19. Emens LA. Breast cancer immunotherapy: facts and hopes. Clin Cancer Res. 2018;24:51120. 
20. Adams S, Gatti-Mays ME, Kalinsky K, Korde LA, Sharon E, Amiri-Kordestani L, et al. Current landscape of immunotherapy in breast cancer: A review. JAMA Oncol. 2019;

21. Nanda R, Chow LQM, Dees EC, Berger R, Gupta S, Geva R, et al. Pembrolizumab in Patients With Advanced Triple-Negative Breast Cancer: Phase Ib KEYNOTE-012 Study. J Clin Oncol. 2016;34:2460-7.

22. Emens LA, Cruz C, Eder JP, Braiteh F, Chung C, Tolaney SM, et al. Long-term Clinical Outcomes and Biomarker Analyses of Atezolizumab Therapy for Patients With Metastatic Triple-Negative Breast Cancer: A Phase 1 Study. JAMA Oncol. 2019;5:74-82.

23. Schmid P, Adams S, Rugo HS, Schneeweiss A, Barrios CH, Iwata H, et al. Atezolizumab and Nab-Paclitaxel in Advanced Triple-Negative Breast Cancer. N Engl J Med. 2018;379:2108-21.

24. Dirix LY, Takacs I, Jerusalem G, Nikolinakos P, Arkenau H-T, Forero-Torres A, et al. Avelumab, an anti-PD-L1 antibody, in patients with locally advanced or metastatic breast cancer: a phase 1b JAVELIN Solid Tumor study. Breast Cancer Res Treat. 2018;167:67186.

25. Loi S, Giobbie-Hurder A, Gombos A, Bachelot T, Hui R, Curigliano G, et al. Pembrolizumab plus trastuzumab in trastuzumab-resistant, advanced, HER2-positive breast cancer (PANACEA): a single-arm, multicentre, phase 1b-2 trial. Lancet Oncol. 2019;20:371-82.

26. Boichard A, Tsigelny IF, Kurzrock R. High expression of PD-1 ligands is associated with kataegis mutational signature and APOBEC3 alterations. Oncoimmunology. 2017;6:e1284719.

27. Boichard A, Pham TV, Yeerna H, Goodman A, Tamayo P, Lippman S, et al. APOBECrelated mutagenesis and neo-peptide hydrophobicity: implications for response to immunotherapy. Oncoimmunology. 2019;8:1550341.

28. Wang S, Jia M, He Z, Liu X-S. APOBEC3B and APOBEC mutational signature as potential predictive markers for immunotherapy response in non-small cell lung cancer. Oncogene. 2018;37:3924-36.

29. Miao D, Margolis CA, Vokes NI, Liu D, Taylor-Weiner A, Wankowicz SM, et al. Genomic correlates of response to immune checkpoint blockade in microsatellite-stable solid tumors. Nat Genet. 2018;50:1271-81.

30. Barroso-Sousa R, Jain E, Cohen O, Kim D, Buendia-Buendia J, Winer E, et al. Prevalence and mutational determinants of high tumor mutation burden in breast cancer. Ann Oncol. 2020;31:387-94.

31. Hollern DP, Xu N, Thennavan A, Glodowski C, Garcia-Recio S, Mott KR, et al. B cells and $\mathrm{T}$ follicular helper cells mediate response to checkpoint inhibitors in high mutation burden mouse models of breast cancer. Cell. 2019;179:1191-1206.e21.

32. Driscoll CB, Schuelke MR, Kottke T, Thompson JM, Wongthida P, Tonne JM, et al. APOBEC3B-mediated corruption of the tumor cell immunopeptidome induces heteroclitic neoepitopes for cancer immunotherapy. Nat Commun. 2020;11:790.

33. Elson A, Leder P. Protein-tyrosine phosphatase epsilon. An isoform specifically expressed in mouse mammary tumors initiated by v-Ha-ras OR neu. J Biol Chem. 1995;270:2611622.

34. Chan K, Roberts SA, Klimczak LJ, Sterling JF, Saini N, Malc EP, et al. An APOBEC3A hypermutation signature is distinguishable from the signature of background mutagenesis by APOBEC3B in human cancers. Nat Genet. 2015;47:1067-72. 
35. Burns MB, Lackey L, Carpenter MA, Rathore A, Land AM, Leonard B, et al. APOBEC3B is an enzymatic source of mutation in breast cancer. Nature. 2013;494:366-70.

36. Akre MK, Starrett GJ, Quist JS, Temiz NA, Carpenter MA, Tutt ANJ, et al. Mutation Processes in 293-Based Clones Overexpressing the DNA Cytosine Deaminase APOBEC3B. PLoS One. 2016;11:e0155391.

37. Nikkilä J, Kumar R, Campbell J, Brandsma I, Pemberton HN, Wallberg F, et al. Elevated APOBEC3B expression drives a kataegic-like mutation signature and replication stressrelated therapeutic vulnerabilities in p53-defective cells. Br J Cancer. 2017;117:113-23.

38. MacMillan AL, Kohli RM, Ross SR. APOBEC3 inhibition of mouse mammary tumor virus infection: the role of cytidine deamination versus inhibition of reverse transcription. $\mathrm{J}$ Virol. 2013;87:4808-17.

39. Landry S, Narvaiza I, Linfesty DC, Weitzman MD. APOBEC3A can activate the DNA damage response and cause cell-cycle arrest. EMBO Rep. 2011;12:444-50.

40. Petljak M, Alexandrov LB, Brammeld JS, Price S, Wedge DC, Grossmann S, et al. Characterizing mutational signatures in human cancer cell lines reveals episodic APOBEC mutagenesis. Cell. 2019;176:1282-1294.e20.

41. Huang X, Wojtowicz D, Przytycka TM. Detecting presence of mutational signatures in cancer with confidence. Bioinformatics. 2018;34:330-7.

42. Li S, Crawford FW, Gerstein MB. Using sigLASSO to optimize cancer mutation signatures jointly with sampling likelihood. Nat Commun. 2020;11:3575.

43. Binnewies M, Roberts EW, Kersten K, Chan V, Fearon DF, Merad M, et al. Understanding the tumor immune microenvironment (TIME) for effective therapy. Nat Med. 2018;24:541-50.

44. Ademmer K, Ebert M, Müller-Ostermeyer F, Friess H, Büchler MW, Schubert W, et al. Effector T lymphocyte subsets in human pancreatic cancer: detection of CD8+CD18+ cells and CD8+CD103+ cells by multi-epitope imaging. Clin Exp Immunol. 1998;112:21-6.

45. Herbst RS, Soria J-C, Kowanetz M, Fine GD, Hamid O, Gordon MS, et al. Predictive correlates of response to the anti-PD-L1 antibody MPDL3280A in cancer patients. Nature. 2014;515:563-7.

46. Beatty GL, Winograd R, Evans RA, Long KB, Luque SL, Lee JW, et al. Exclusion of T cells from pancreatic carcinomas in mice is regulated by ly6c(low) F4/80(+) extratumoral macrophages. Gastroenterology. 2015;149:201-10.

47. Mlecnik B, Bindea G, Angell HK, Maby P, Angelova M, Tougeron D, et al. Integrative analyses of colorectal cancer show immunoscore is a stronger predictor of patient survival than microsatellite instability. Immunity. 2016;44:698-711.

48. Hosoi A, Takeda K, Nagaoka K, Iino T, Matsushita H, Ueha S, et al. Increased diversity with reduced "diversity evenness" of tumor infiltrating T-cells for the successful cancer immunotherapy. Sci Rep. 2018;8:1058.

49. Nik-Zainal S, Van Loo P, Wedge DC, Alexandrov LB, Greenman CD, Lau KW, et al. The life history of 21 breast cancers. Cell. 2012;149:994-1007.

50. Jamal-Hanjani M, Wilson GA, McGranahan N, Birkbak NJ, Watkins TBK, Veeriah S, et al. Tracking the Evolution of Non-Small-Cell Lung Cancer. N Engl J Med. 2017;376:2109-21.

51. McGranahan N, Favero F, de Bruin EC, Birkbak NJ, Szallasi Z, Swanton C. Clonal status of actionable driver events and the timing of mutational processes in cancer evolution. Sci Transl Med. 2015;7:283ra54. 
1000

1001

1002

1003

1004

1005

1006

1007

1008

1009

1010

1011

1012

1013

1014

1015

1016

1017

1018

1019

1020

1021

1022

1023

1024

1025

1026

1027

1028

1029

1030

1031

1032

1033

1034

1035

1036

1037

1038

1039

1040

1041

1042

1043

1044

1045

52. Jia Q, Wu W, Wang Y, Alexander PB, Sun C, Gong Z, et al. Local mutational diversity drives intratumoral immune heterogeneity in non-small cell lung cancer. Nat Commun. 2018;9:5361.

53. Rosenthal R, Cadieux EL, Salgado R, Bakir MA, Moore DA, Hiley CT, et al. Neoantigendirected immune escape in lung cancer evolution. Nature. 2019;567:479-85.

54. Wolf Y, Bartok O, Patkar S, Eli GB, Cohen S, Litchfield K, et al. UVB-Induced Tumor Heterogeneity Diminishes Immune Response in Melanoma. Cell. 2019;179:219-235.e21.

55. McGranahan N, Furness AJS, Rosenthal R, Ramskov S, Lyngaa R, Saini SK, et al. Clonal neoantigens elicit $\mathrm{T}$ cell immunoreactivity and sensitivity to immune checkpoint blockade. Science. 2016;351:1463-9.

56. Bindea G, Mlecnik B, Tosolini M, Kirilovsky A, Waldner M, Obenauf AC, et al. Spatiotemporal dynamics of intratumoral immune cells reveal the immune landscape in human cancer. Immunity. 2013;39:782-95.

57. Faruki H, Mayhew GM, Serody JS, Hayes DN, Perou CM, Lai-Goldman M. Lung adenocarcinoma and squamous cell carcinoma gene expression subtypes demonstrate significant differences in tumor immune landscape. J Thorac Oncol. 2017;12:943-53.

58. Savas P, Salgado R, Denkert C, Sotiriou C, Darcy PK, Smyth MJ, et al. Clinical relevance of host immunity in breast cancer: from TILs to the clinic. Nat Rev Clin Oncol. 2016;13:228-41.

59. Cimino-Mathews A, Thompson E, Taube JM, Ye X, Lu Y, Meeker A, et al. PD-L1 (B7H1) expression and the immune tumor microenvironment in primary and metastatic breast carcinomas. Hum Pathol. 2016;47:52-63.

60. Glaser AP, Fantini D, Wang Y, Yu Y, Rimar KJ, Podojil JR, et al. APOBEC-mediated mutagenesis in urothelial carcinoma is associated with improved survival, mutations in DNA damage response genes, and immune response. Oncotarget. 2018;9:4537-48.

61. Raynaud F, Mina M, Tavernari D, Ciriello G. Pan-cancer inference of intra-tumor heterogeneity reveals associations with different forms of genomic instability. PLoS Genet. 2018; 14:e1007669.

62. Ellsworth RE, Ellsworth DL, Patney HL, Deyarmin B, Love B, Hooke JA, et al. Amplification of HER2 is a marker for global genomic instability. BMC Cancer. 2008;8:297.

63. Takahashi H, Asaoka M, Yan L, Rashid OM, Oshi M, Ishikawa T, et al. Biologically Aggressive Phenotype and Anti-cancer Immunity Counterbalance in Breast Cancer with High Mutation Rate. Sci Rep. 2020;10:1852.

64. Smid M, Rodríguez-González FG, Sieuwerts AM, Salgado R, Prager-Van der Smissen WJC, Vlugt-Daane $\mathrm{M}$ van der, et al. Breast cancer genome and transcriptome integration implicates specific mutational signatures with immune cell infiltration. Nat Commun. 2016;7:12910.

65. Faden DL, Ding F, Lin Y, Zhai S, Kuo F, Chan TA, et al. APOBEC mutagenesis is tightly linked to the immune landscape and immunotherapy biomarkers in head and neck squamous cell carcinoma. Oral Oncol. 2019;96:140-7.

66. McDonald K-A, Kawaguchi T, Qi Q, Peng X, Asaoka M, Young J, et al. Tumor Heterogeneity Correlates with Less Immune Response and Worse Survival in Breast Cancer Patients. Ann Surg Oncol. 2019;26:2191-9.

67. Chen Z, Wen W, Bao J, Kuhs KL, Cai Q, Long J, et al. Integrative genomic analyses of APOBEC-mutational signature, expression and germline deletion of APOBEC3 genes, and 
1064

1065

1066

1067

1068

1069

1070

1071

1072

1073

1074

1075

1076

1077

1078

1079

1080

1081

1082

1083

1084

1085

1086

1087

1088

1089

1090

1091

immunogenicity in multiple cancer types. BMC Med Genomics. 2019;12:131.

68. Adolph MB, Love RP, Feng Y, Chelico L. Enzyme cycling contributes to efficient induction of genome mutagenesis by the cytidine deaminase APOBEC3B. Nucleic Acids Res. 2017;45:11925-40.

69. Chédin F. Nascent Connections: R-Loops and Chromatin Patterning. Trends Genet. 2016;32:828-38.

70. Mabe NW, Fox DB, Lupo R, Decker AE, Phelps SN, Thompson JW, et al. Epigenetic silencing of tumor suppressor Par-4 promotes chemoresistance in recurrent breast cancer. $\mathrm{J}$ Clin Invest. 2018;128:4413-28.

71. Dobin A, Davis CA, Schlesinger F, Drenkow J, Zaleski C, Jha S, et al. STAR: ultrafast universal RNA-seq aligner. Bioinformatics. 2013;29:15-21.

72. Liao Y, Smyth GK, Shi W. featureCounts: an efficient general purpose program for assigning sequence reads to genomic features. Bioinformatics. 2014;30:923-30.

73. Love MI, Huber W, Anders S. Moderated estimation of fold change and dispersion for RNA-seq data with DESeq2. Genome Biol. 2014;15:550-550.

74. Ashburner M, Ball CA, Blake JA, Botstein D, Butler H, Cherry JM, et al. Gene Ontology: tool for the unification of biology. Nat Genet. 2000;25:25-9.

75. The Gene Ontology Consortium. The Gene Ontology Resource: 20 years and still GOing strong. Nucleic Acids Res. 2019;47:D330-8.

76. Subramanian A, Tamayo P, Mootha VK, Mukherjee S, Ebert BL, Gillette MA, et al. Gene set enrichment analysis: a knowledge-based approach for interpreting genome-wide expression profiles. Proc Natl Acad Sci USA. 2005;102:15545-50.

77. Mootha VK, Lindgren CM, Eriksson K-F, Subramanian A, Sihag S, Lehar J, et al. PGC1alpha-responsive genes involved in oxidative phosphorylation are coordinately downregulated in human diabetes. Nat Genet. 2003;34:267-73.

78. Newman AM, Steen CB, Liu CL, Gentles AJ, Chaudhuri AA, Scherer F, et al. Determining cell type abundance and expression from bulk tissues with digital cytometry. Nat Biotechnol. 2019;37:773-82.

79. Bolotin DA, Poslavsky S, Mitrophanov I, Shugay M, Mamedov IZ, Putintseva EV, et al. MiXCR: software for comprehensive adaptive immunity profiling. Nat Methods. 2015;12:380-1.

80. Grossman RL, Heath AP, Ferretti V, Varmus HE, Lowy DR, Kibbe WA, et al. Toward a shared vision for cancer genomic data. N Engl J Med. 2016;375:1109-12.

81. Colaprico A, Silva TC, Olsen C, Garofano L, Cava C, Garolini D, et al. TCGAbiolinks: an R/Bioconductor package for integrative analysis of TCGA data. Nucleic Acids Res. 2016;44:e71.

82. Anders S, Huber W. Differential expression analysis for sequence count data. Genome Biol. 2010;11:R106.

83. Law CW, Chen Y, Shi W, Smyth GK. voom: Precision weights unlock linear model analysis tools for RNA-seq read counts. Genome Biol. 2014;15:R29.

84. Robinson MD, McCarthy DJ, Smyth GK. edgeR: a Bioconductor package for differential expression analysis of digital gene expression data. Bioinformatics. 2010;26:139-40.

85. Ritchie ME, Phipson B, Wu D, Hu Y, Law CW, Shi W, et al. limma powers differential expression analyses for RNA-sequencing and microarray studies. Nucleic Acids Res. 2015;43:e47.

86. Benjamin DI, Sato T, Lichtenstein L, Stewart C, Getz G, Cibulskis K. Calling Somatic 
bioRxiv preprint doi: https://doi.org/10.1101/2021.02.13.431068; this version posted February 14, 2021. The copyright holder for this preprint

(which was not certified by peer review) is the author/funder, who has granted bioRxiv a license to display the preprint in perpetuity. It is made available under aCC-BY-NC-ND 4.0 International license.

SNVs and Indels with Mutect2. BioRxiv. 2019;

1093 87. Dabney AR. Classification of microarrays to nearest centroids. Bioinformatics.

1094 2005;21:4148-54. 
Figure 1 bioRxiv preprint doi: https://doi.org/10.1101/2021.02.13.431068; this version posted February 14,2021 . The copyright holder for this preprint A
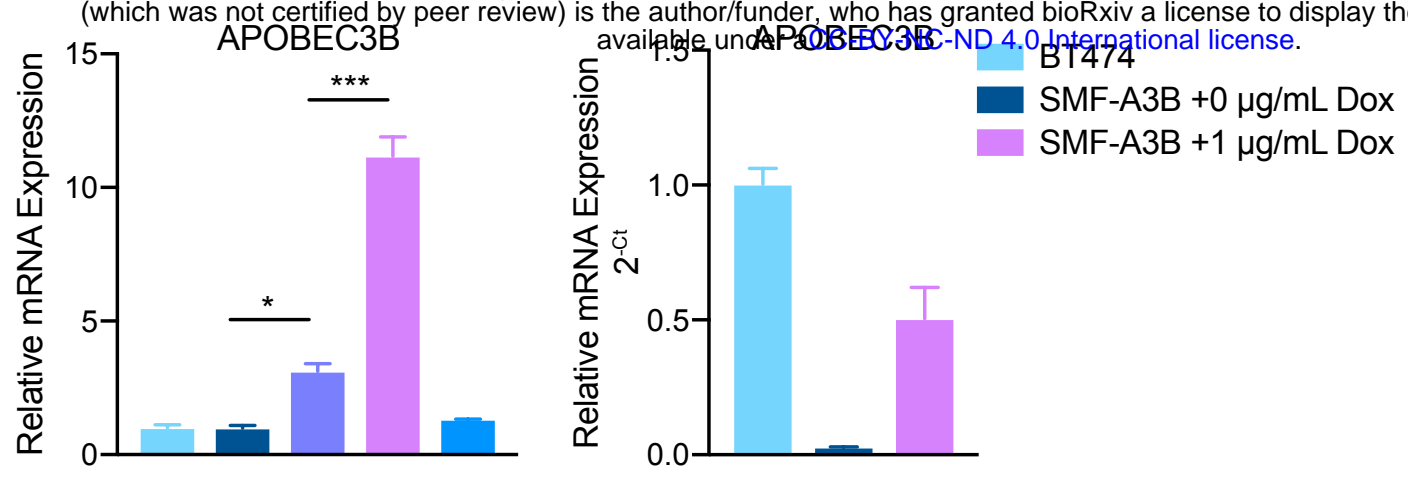

Dox $(\mu \mathrm{g} / \mathrm{mL}): \quad 0 \quad 0.010 .1 \quad 1 \quad 1+/-$

B

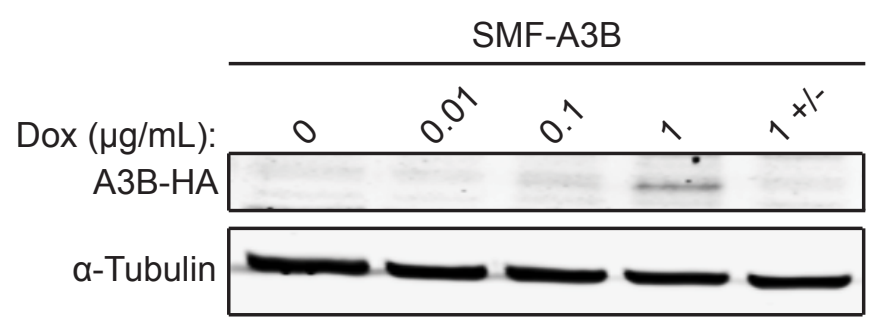

D

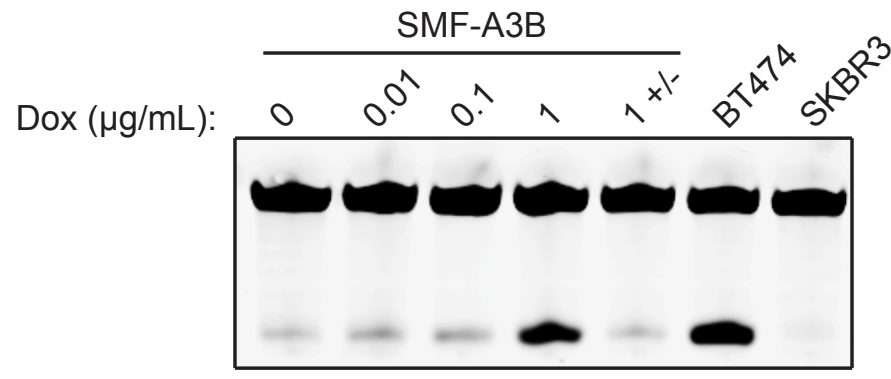

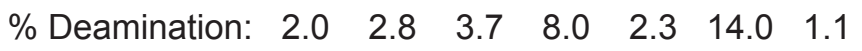

C

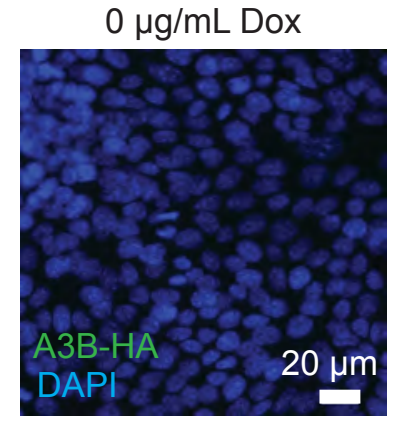

E

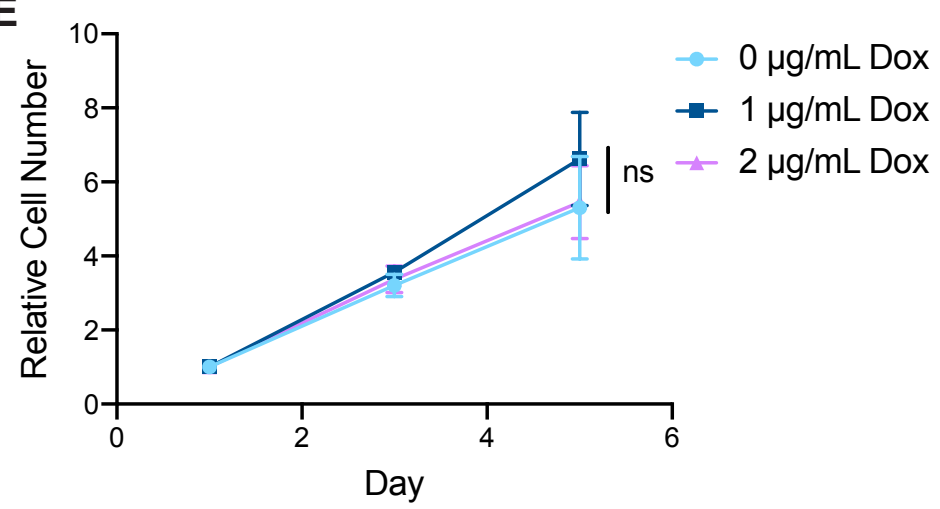

G

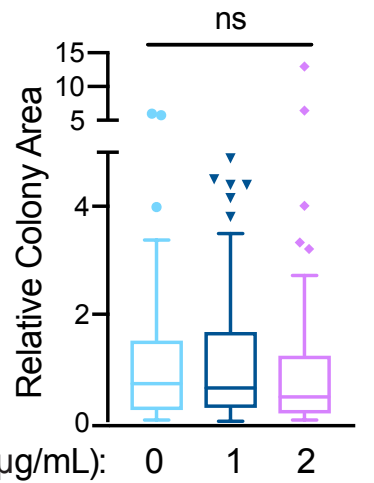

$1 \mu \mathrm{g} / \mathrm{mL}$ Dox
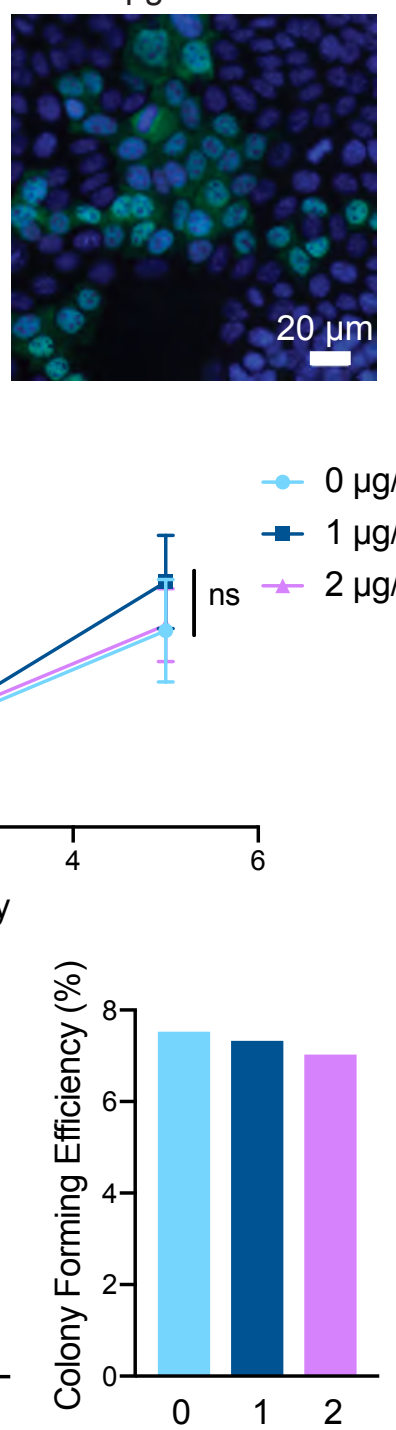

Figure 1: SMF-A3B cells express titratable and reversible APOBEC3B without loss in cell viability. (A) qRTPCR of A3B gene expression in SMF-A3B cells treated with the indicated concentrations of dox for 2 days. $1+/-$ indicates treatment with $1 \mu \mathrm{g} / \mathrm{mL}$ of dox for 2 days, then removal of dox for 2 days. Left: A3B expression relative to 0 $\mu \mathrm{g} / \mathrm{mL}$ dox condition. Right: A3B expression relative to BT474 cells. Data are representative of 2 independent experiments. Results show 3 biological replicates and error bars depict mean \pm SEM. Significance was determined using a one-way ANOVA and Tukey's multiple comparisons test. (B) Western blot of HA-tagged A3B in SMF-A3B cells treated with dox as in (A). (C) Immunofluorescence staining for the HA epitope in SMF-A3B cells treated with dox as in (A), showing nuclear localization of HA-A3B. Blue channel is DAPI and green channel is HA. (D) In vitro cytidine deaminase activity assay of SMF-A3B cells treated with dox as in (A). The APOBEC-high human cell line BT474 and the A3B-null human cell line SKBR3 are shown as controls. (E) CellTiter-Glo assay showing growth curves of SMFA3B cells treated with the indicated concentration of dox. Results are shown as mean \pm SD of 3 replicates. Statistical significance was determined by two-way ANOVA. (F) Clonogenic assay of SMF-A3B cells cultured with dox for 2 weeks to measure long term survival. Colonies were stained with crystal violet. (G) Quantification of clonogenic assay in $(F)$. Left: Boxplots depicting the relative colony area. Statistical significance was determined using a one-way ANOVA. Right: Colony forming efficiency in each condition. ns $p>0.05,{ }^{*} p<0.05,{ }^{* *} p<0.01,{ }^{* * *} p<0.001$ 


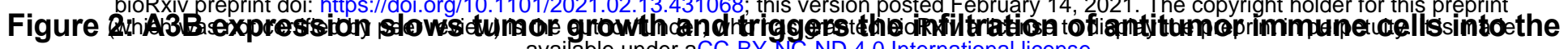

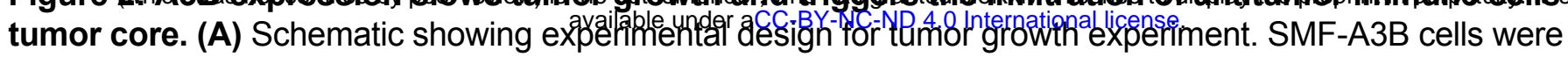
orthotopically implanted in the mammary gland of mice. The APOBEC cohort was administered dox in the drinking water and control cohort was administered normal drinking water until endpoint. (B) Left: tumor volume of control $(n=20)$ and APOBEC tumors $(n=20)$ in wildtype mice. Statistical significance was determined by two-way repeated-measures ANOVA. Right: tumor mass (g) of control and APOBEC tumors at endpoint. Statistical significance was determined by unpaired Student's t-test. Error bars denote mean \pm SEM. Data is representative of 2 independent experiments. (C) The frequency of immune cell types, expressed as a percentage of total CD45+ cells, in control $(n=6)$ and APOBEC $(n=6)$ tumors as determined by flow cytometry. (D) Flow cytometry quantification of immune cells in control $(n=6)$ and APOBEC ( $n=6)$ tumors. Leukocytes, CD8+ T cells, CD4+ T cells, CD103+ dendritic cells (DCs), and macrophages are represented as the percentage of total live cells. T regulatory cells (Tregs) and type-2 T helper (Th2) cells are represented as the percentage of total CD4+ T cells. Statistical significance was determined by unpaired Student's t-test. Error bars denote mean \pm SEM. (E-F) Representative flow cytometry plots $(E)$ and quantification (F) of staining for IFN , Granzyme B, PD-1 and PD-L1 in control $(n=6)$ and APOBEC $(n=6)$ tumors. Statistical significance was determined by unpaired Student's t-test. Error bars denote mean \pm SEM. (G) Pearson correlation between immune cell frequency and mean tumor volume ( $\mathrm{mm} 3$ ) in control (light blue) and APOBEC (dark blue) tumors. Only significant correlations are shown, and the $r$ squared values are indicated. $(\mathbf{H})$ Immunohistochemistry $(\mathrm{IHC})$ staining for the T cell marker CD3 in control and APOBEC tumors. The top image is a tiled scan of the whole tumor, and the bottom image is a representative region in the tumor core. (I) Quantification of CD3 staining for control $(n=4)$ and APOBEC $(n=4)$ tumors. Four fields of view were imaged for each tumor. Boxplots show the median percentage of CD3+ cells with minimum and maximum whiskers. Statistical significance was determined by unpaired Student's t test with Welch's correction. ${ }^{*} p<0.05,{ }^{* *} p<$ $0.01,{ }^{* * *} p<0.001$ 


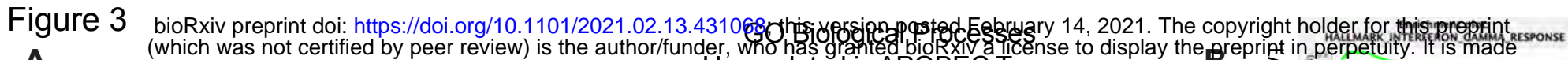
available undertipregulatediinAPQB EAtohtumersse.

negative regulation of $\mathrm{T}$ cell mediated immunity

regulation of $\mathrm{T}$ cell cytokine production

positive regulation of lymphocyte chemotaxis positive regulation of $\mathrm{T}$ cell mediated cytotoxicity positive regulation of $\mathrm{T}$ cell proliferation regulation of $\mathrm{T}$ cell migration phagocytosis, recognition positive regulation of $B$ cell activation phagocytosis, engulfment complement activation, classical pathway positive regulation of $\mathrm{T}$ cell differentiation

$\mathrm{T}$ cell activation defense response to bacterium

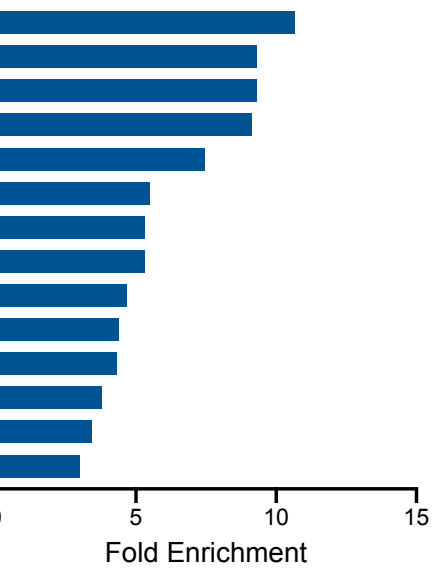

C

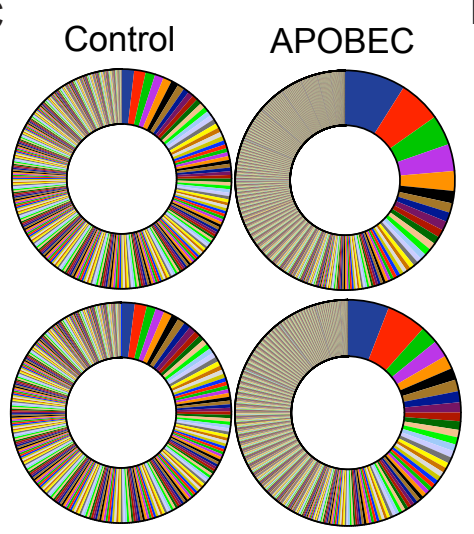

D

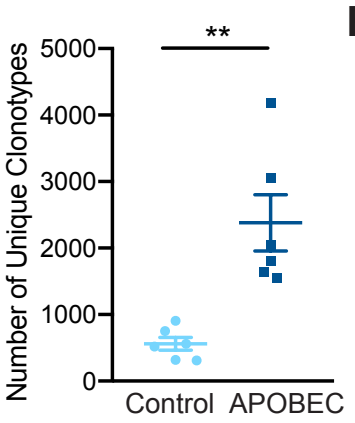

E

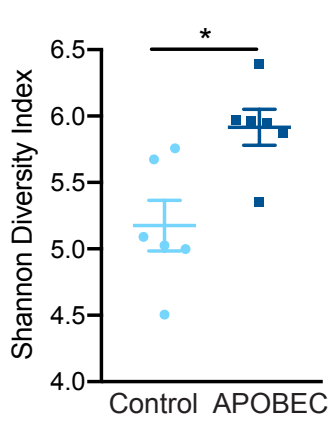

NES $=2.98$

FDR q-value $=0$

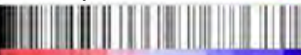

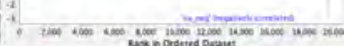
Enrichment ploc: HAUMAgK OMA PSPA

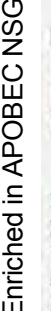

NES $=1.67$

FDR q-value $=0.0016$

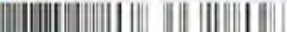

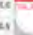

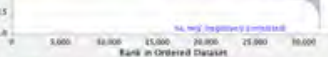

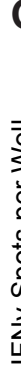

\section{Control + algG}

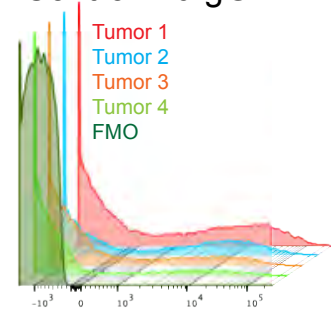

MHC-I

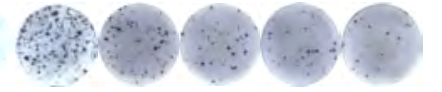

H - Control + algG, $n=14$

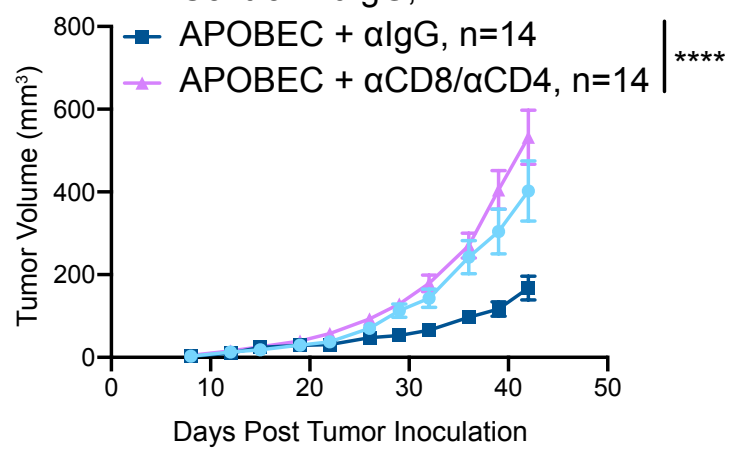

J
$\mathbf{F}$

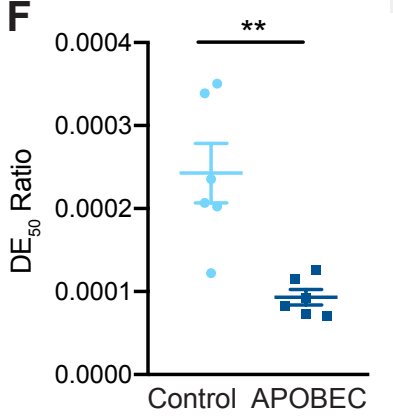

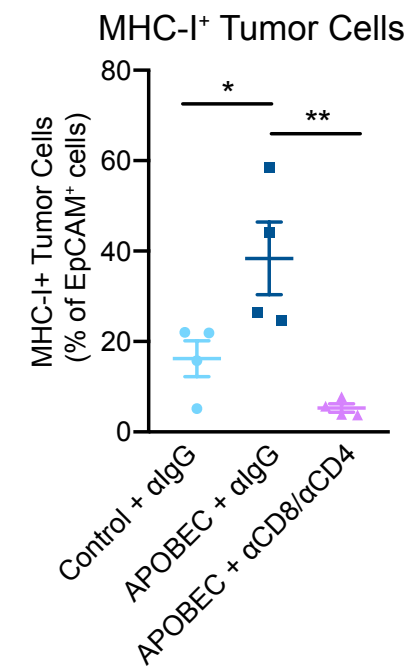


bioRxiv preprint doi: https://doi.org/10.1101/2021.02.13.431068; this version posted February 14, 2021. The copyright holder for this preprint

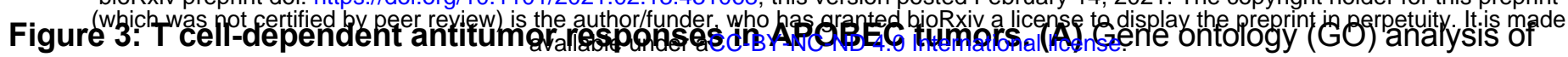
differentially expressed genes between control and APOBEC tumors in immunocompetent mice. Bar graph shows the fold enrichment of select GO biological processes that were significantly enriched in APOBEC tumors $(n=6)$ compared to control tumors $(n=6)(F D R<0.05$, Fisher's test). All significantly upregulated biological process $G O$ terms are shown in Supplementary Fig. S6A. (B) Gene set enrichment analysis (GSEA) of differentially expressed genes between control and APOBEC tumors. Representative gene sets enriched in APOBEC tumors in immunocompetent mice (top) or immunodeficient mice (bottom) are shown. Normalized Enrichment Scores (NES) and FDR q-values are shown. All significantly enriched gene sets are shown in Supplementary Fig. S6B, C. (C) T cell receptor (TCR) sequencing from control $(n=6)$ and APOBEC tumors $(n=6)$ from wildtype mice. Pie charts show unique TCR clonotypes ranked by abundance in two control and two APOBEC tumors. (D) Quantification of the total number of unique clonotypes in control and APOBEC tumors ( $n=6$ per cohort). (E) The Shannon diversity index of the TCR repertoire in control and APOBEC tumors. (F) TCR diversity evenness 50 (DE50) ratios in control and APOBEC tumors. DE50 ratio is calculated by the number of clonotypes composing the top $50 \%$ of total read counts divided by the total number of read counts. Error bars in (C-E) denote mean \pm SEM. Statistical significance was determined by unpaired Student's $t$ test in (D) and unpaired Student's $t$ test with Welch's correction in (C) and (E). (G) Representative ELISpot images and quantification of the number of IFNy spots per well for each condition. NS, naïve splenocytes from a non-tumor-bearing mouse. ConA, concanavalin A model antigen. Error bars denote mean \pm SD from 4 technical replicates per condition. (H) Tumor volume (mm3) over time for control tumors treated with isotype control antibody $(n=14)$ and APOBEC tumors treated with isotype control $(n=14)$ or $\alpha C D 8$ and $\alpha C D 4$ depletion antibodies $(n=14)$ in wildtype mice. Error bars denote mean \pm SEM. Statistical significance was determined by two-way repeated-measures ANOVA and Tukey's multiple comparisons test. (I) Flow cytometry histograms showing MHC-I expression on EpCAM+ tumor cells from tumors in $(\mathrm{H})$. (J) Quantification of MHC-I+ cells, expressed as a percentage of EpCAM+ cells, in tumors ( $n=4$ per cohort) from $(H)$. Error bars denote mean \pm SEM. Statistical significance was determined by one-way ANOVA and Tukey's multiple comparisons test. ${ }^{*} p<0.05,{ }^{* *} p<0.01,{ }^{* * * *} p<0.0001$ 
Figure 4 bioRxiv preprint doi: https://doi.org/10.1101/2021.02.13.431068; this version posted February 14, 2021. The copyright holder for this preprint (which was not certified by peer review) is the author/funder, who has granted bioRxiv a license to display the preprint in perpetuity. It is made
available under aCC-BY-NC-ND 4.0 International license.

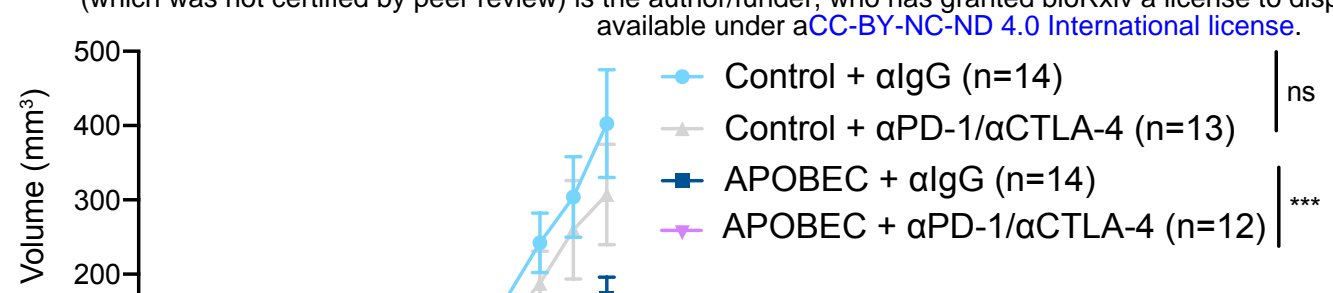

B

B Control + algG

Control $+\alpha P D-1 / \alpha C T L A-4$ (CR/PR: 1 of 13)

- $\mathrm{APOBEC}+$ algG

APOBEC + aPD-1/aCTLA-4 (CR/PR: 7 of 11)

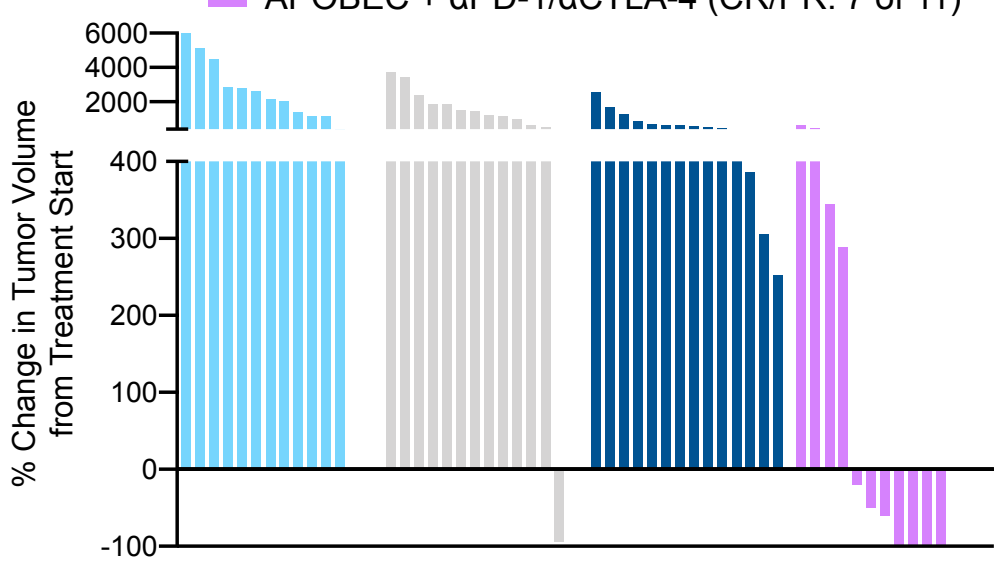

Figure 4: APOBEC tumors are sensitive to combination anti-PD-1/anti-CTLA4 immune checkpoint blockade. (A) Tumor volume $(\mathrm{mm} 3)$ over time for control and APOBEC tumors treated with isotype control or aPD-1/aCTLA-4 antibodies. Arrow indicates the treatment start. Error bars denote mean \pm SEM. Statistical significance was determined by two-way repeated-measures ANOVA and Tukey's multiple comparisons test. (B) Change in tumor volume from treatment start for palpable tumors until endpoint. Each bar denotes an individual tumor. CR, complete response; PR, partial response. ns $p>0.05,{ }^{* \star *} p<0.001$ 
Figure 5 bioRxiv preprint doi: https://doi.org/10.1101/2021.02.13.431068; this version posted February 14, 2021. The copyright holder for this preprint

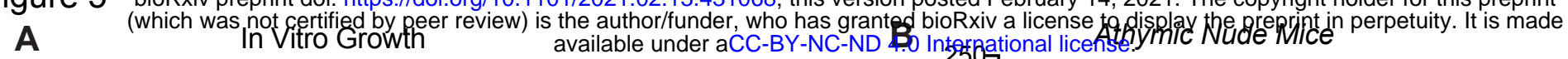
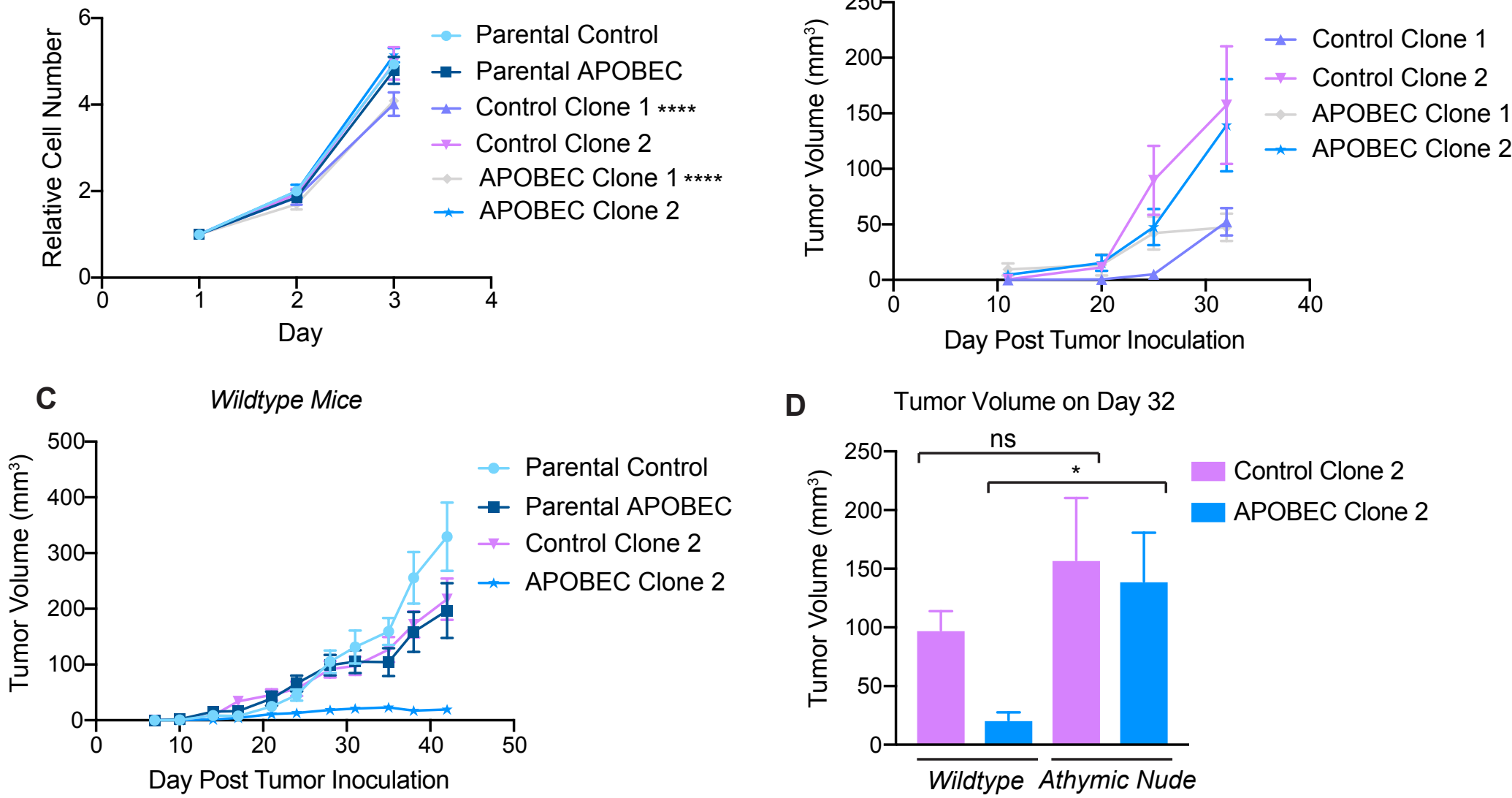

D Tumor Volume on Day 32

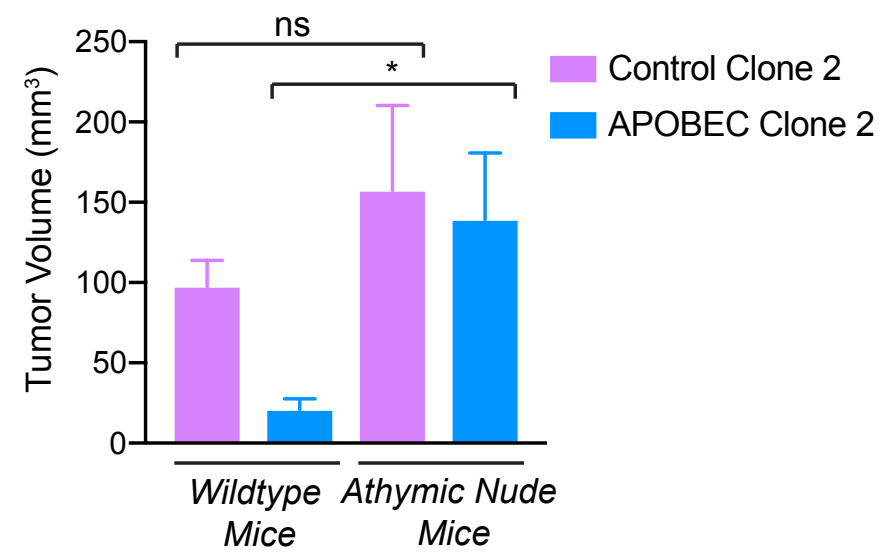

Figure 5: Complete immune-mediated suppression of clonal APOBEC tumor growth. (A) In vitro growth curves of single-cell clones derived from control SMF-A3B cells (control) or SMF-A3B cells treated with dox for 2 weeks (APOBEC). The growth curves of the polyclonal parental cells (parental control or parental APOBEC) are shown as a control. Error bars denote mean \pm SD of 4 replicates. Control Clone 1 and APOBEC Clone 1 proliferate more slowly than the parental control cells, as determined by two-way ANOVA and Dunnett's multiple comparison test. (B) Tumor volume $(\mathrm{mm} 3)$ over time for control and APOBEC clones injected in the mammary gland of athymic nude mice. Error bars denote mean \pm SEM. (C) Tumor volume $(\mathrm{mm} 3)$ over time for control and APOBEC clones, as well as the corresponding polyclonal parental populations, injected in the mammary gland of immunocompetent wildtype mice. Error bars denote mean \pm SEM. (D) Comparison of tumor volume on day 32 between clones grown in immunocompromised nude mice from (B) and immunocompetent wildtype mice from (C). Error bars denote mean \pm SEM and statistical significance was determined by one-way ANOVA and Sidak's multiple comparisons test. ns $p>$ $0.05,{ }^{*} p<0.05,{ }^{* * * *} p<0.0001$ 
Figure 6 bioRxiv preprint doi: https://doi.org/10.1101/2021.02.13.431068; this version posted February 14, 2021. The copyright holder for this preprint A (which was not certified by peer review) is the author/funder, who has granted bioRxiv a license to display the preprint in perpetuity. It is made

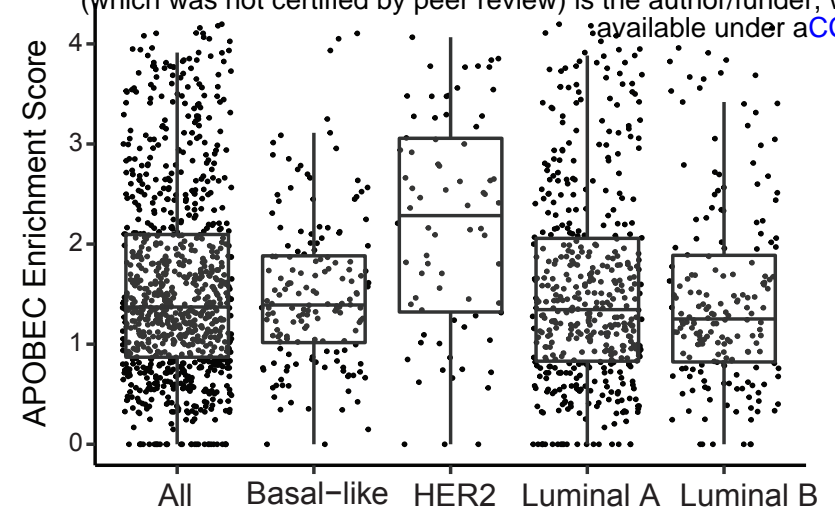

B

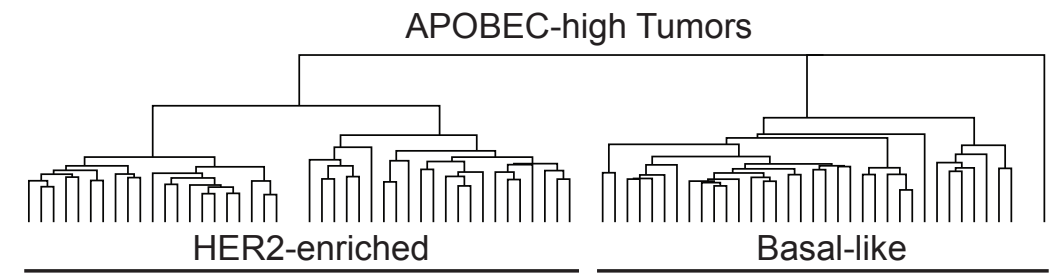

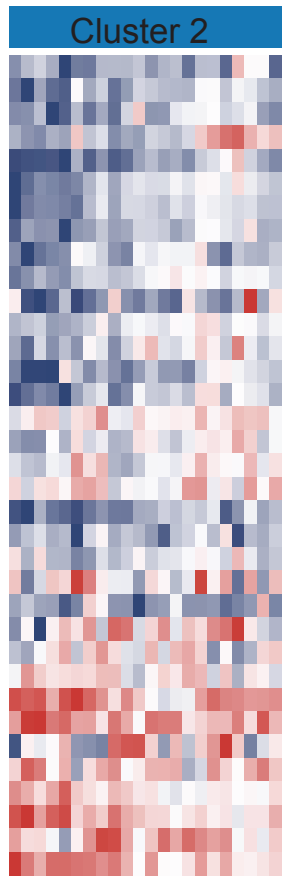

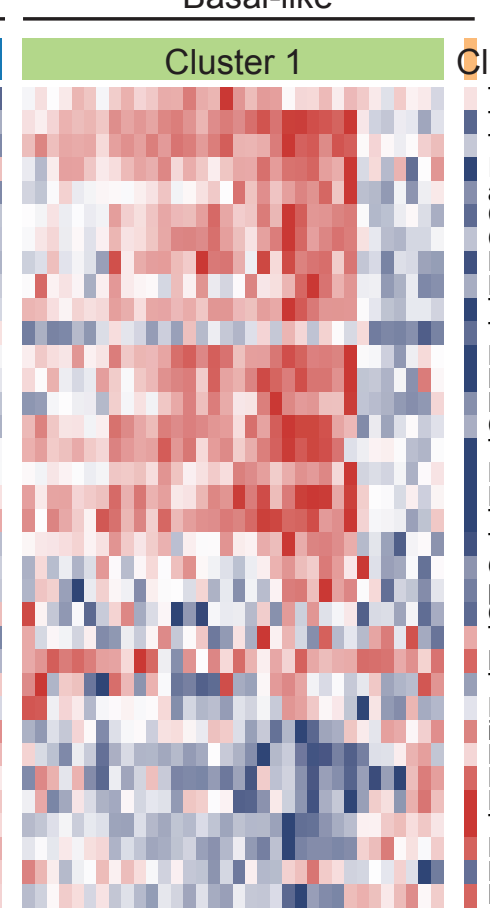

C Correlations of APOBEC Enrichment

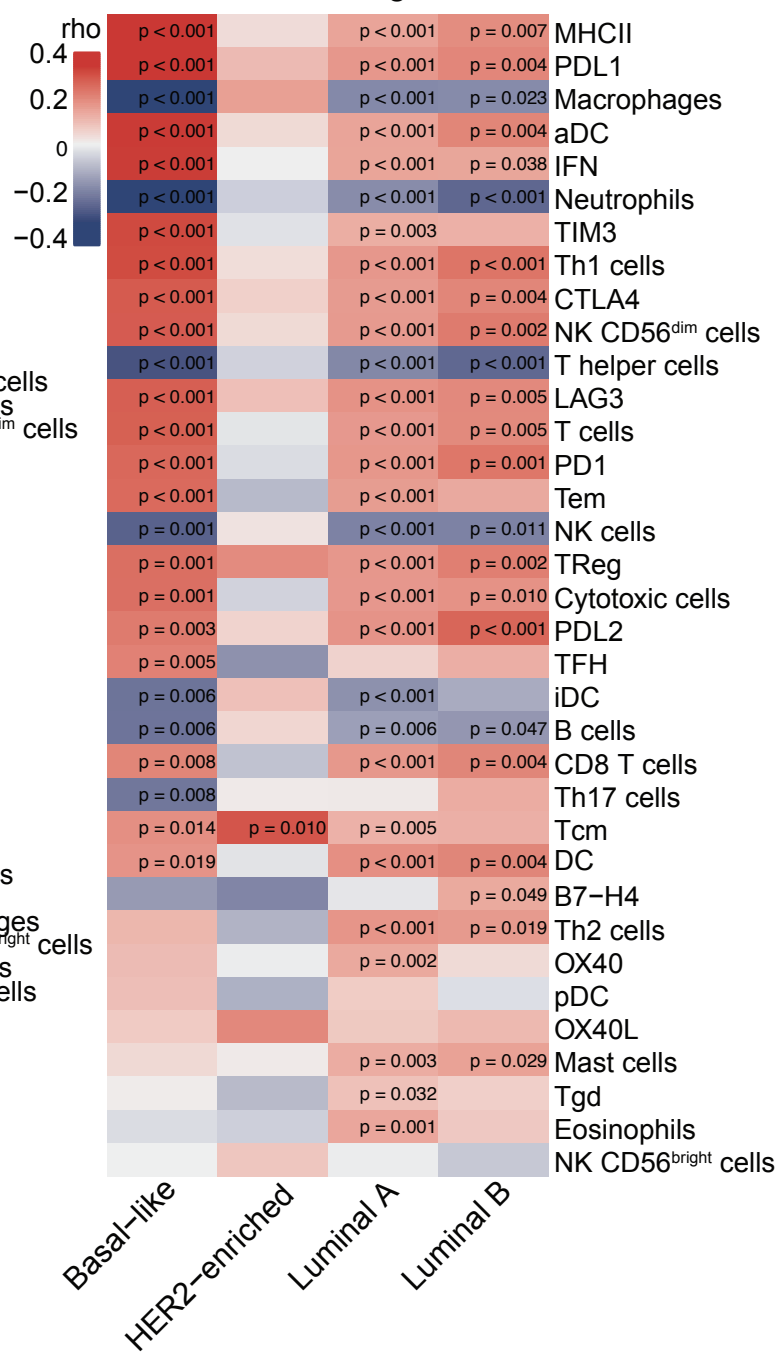
and Immune Signatures

$-0.2$
APOBEC-high Cluster 1 APOBEC-high Cluster 2 APOBEC-low Cluster 1 APOBEC-low Cluster 2
Cluster 2

Th2 cells TFH IFN Cytotoxic cells CD8 T cells DC $T$ cells Igd MHCII LAG3 CTLA4 TReg $\mathrm{PDL} 1$
$\mathrm{PDL} 2$ TIM3 Tem pDC Th17 B7-H4 Eosinophils iDC Macrophage NK CD $56^{6}$ ight Neutrophils Thelper cells NK cells Mast cells $B$ cells
D

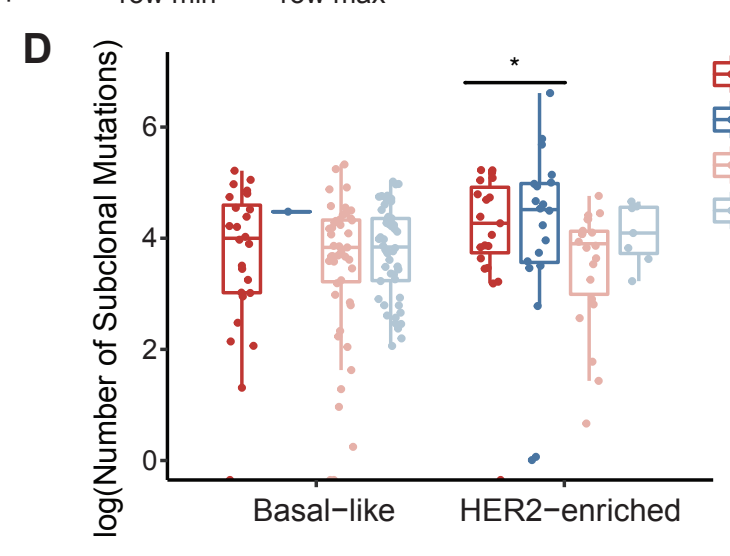

Relative

Expression row min row max 


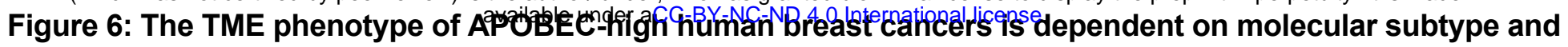
the number of subclonal mutations. (A) APOBEC enrichment score calculated from whole-exome sequencing (WES) data for all TCGA breast cancer samples. APOBEC enrichment score of 2 or higher delineates APOBEC-high tumors. Boxplots show 25th percentile, median, and 75th percentile, while whiskers show minimum and maximum values excluding outliers. (B) Heatmap showing the relative expression of immune cell gene signatures from TCGA RNA-seq data in APOBEC-high tumors, grouped by breast cancer subtype. Columns are individual patient tumors and rows are different immune cell gene signatures. Legend shows colors corresponding to relative expression levels (red, row max; blue, row min). Hierarchical clustering segregated tumors into 2 main clusters in the HER2-enriched subtype and 2 clusters in the basal-like subtype. (C) Heatmap showing correlation Spearman rho values between APOBEC enrichment score and immune gene signatures for each molecular subtype of breast cancer. p-values are shown and legend shows colors corresponding to rho value (red, immune signature positively correlated with APOBEC enrichment score; blue, immune signature negatively correlated with APOBEC enrichment score). (D) The number of subclonal mutations (from Raynaud et al.) in APOBEC-high clusters depicted in (B) and APOBEC-low clusters depicted in Supplementary Figure S9. APOBEC-high HER2-enriched tumors in cluster 2 had more subclonal mutations than tumors in cluster 1 . Boxplots show 25th percentile, median, and 75th percentile, while whiskers show minimum to maximum values excluding outliers. Statistical significance was determined one-way ANOVA and Sidak's multiple comparisons test. * $p<0.05$. MHC-II, major histocompatibility complex class II antigen presentation; IFN, interferon signaling pathway; Th1 cells, type-1 T helper cells; Th2 cells, type-2 T helper cells; Tgd, T gamma delta cells; Treg, T regulatory cells; Tem, T effector memory cells; Tcm, T central memory cells; TFH, T follicular helper cells; Th17, Thelper 17 cells; DC, dendritic cells; aDC, activated dendritic cells; iDC, immature dendritic cells; pDC, plasmacytoid dendritic cells; CD56dim NK cells, CD56 dim natural killer cells; CD56bright NK cells, CD56 bright natural killer cells. 
A

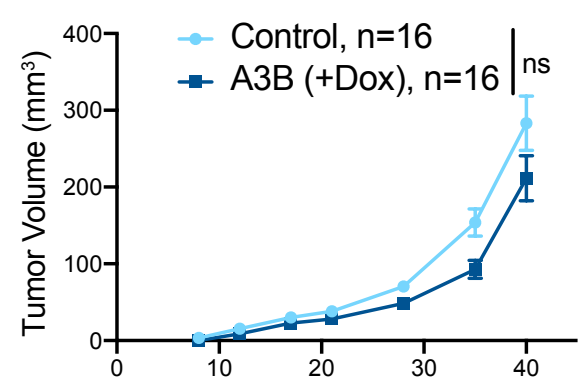

Days Post Tumor Inoculation available under aCC-BY-NC-ND 4.0 International license.

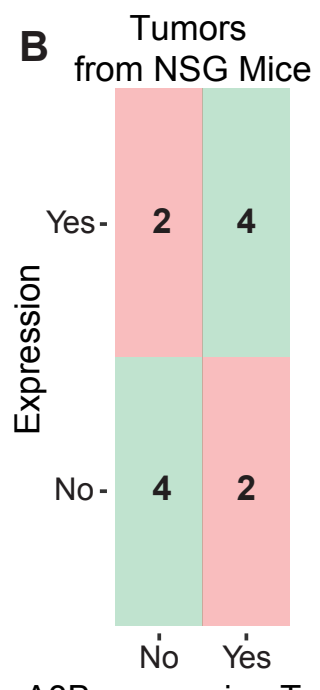

A3B-expressing Tumor

C

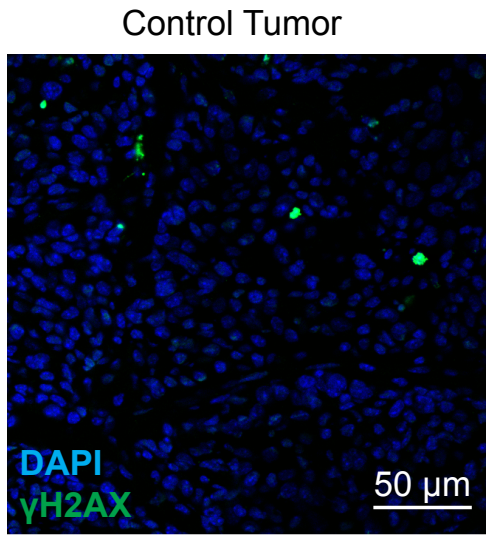

$\mathbf{E}$

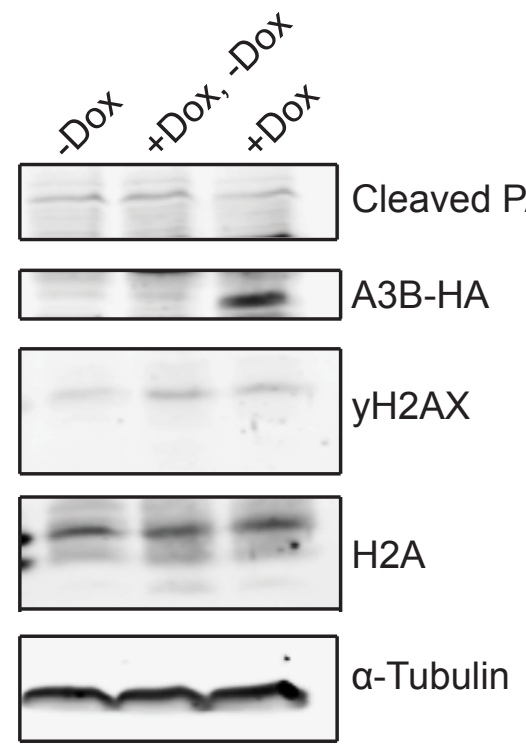

APOBEC Tumor

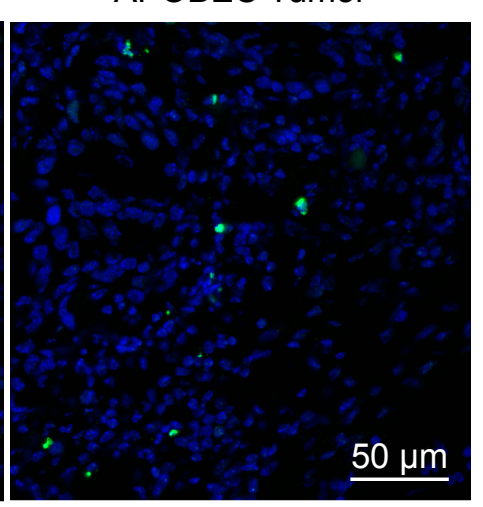

D

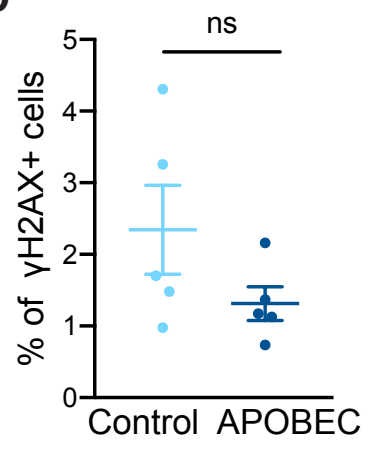

Supplementary Figure S1: A3B expression does not alter tumor growth in immunodeficient mice and induces an APOBEC mutational gene expression signature without activating the DNA damage response. (A) Tumor volume $(\mathrm{mm} 3)$ over time for control $(n=16)$ and A3B-expressing tumors (+dox in drinking water; $n=16$ ) growing in NSG mice. Error bars denote mean \pm SEM. Statistical significance was determined by two-way repeated-measures ANOVA and Tukey's multiple comparisons test with the same control cohort as in Supplementary Fig. S5J. (B) Confusion matrix of 10 gene predictor applied to sample of 12 mouse tumors in NSG mice (6 A3B-expressing tumors and 6 control tumors). Squares in red (upper left and bottom right) denote incorrect classifications and squares in green (upper right and bottom left) represent correct classifications. Sensitivity and specificity were both $66 \%$. (C-D) Immunofluorescence staining for $\mathrm{YH} 2 \mathrm{AX}$ on control and APOBEC tumors growing in wildtype mice (see Figure 2B). Representative images are shown in (C) and quantification of $\mathrm{yH} 2 \mathrm{AX}+$ foci (number of foci/number of cells per field of view) is shown in (D). Five tumors per cohort were analyzed and 8 fields of view were averaged per tumor. DAPI is in blue and $\mathrm{YH} 2 \mathrm{AX}$ is in green. Error bars denote mean \pm SEM and statistical significance was determined by unpaired Student's t-test. (E) Western blot analysis of HA-epitope tagged A3B, $\mathrm{YH} 2 \mathrm{AX}$, and cleaved PARP in SMF-A3B cells treated with or without dox for 2 weeks. $\alpha$-Tubulin and histone $\mathrm{H} 2 \mathrm{~A}$ are shown as loading controls. ns $>0.05$ 
Supplemebtakx Frighteos/2tps://doi.org/10.1101/2021.02.13.431068; this version posted February 14, 2021. The copyright holder for this preprint A (which was not certified by peer review) is the author/funder, who has granted bioRxiv a license to display the preprint in perpetuity. It is made

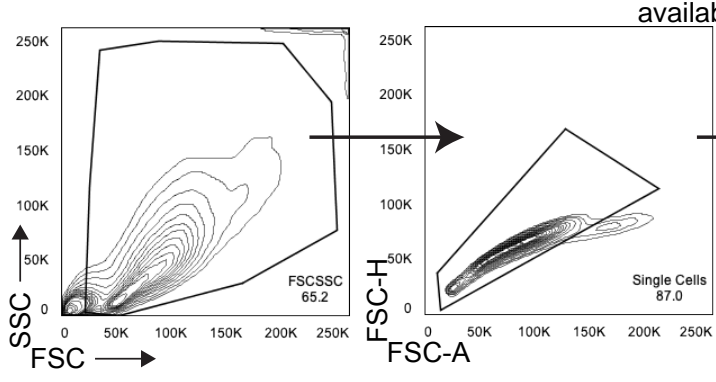
ailable under aCC-BY-NC-ND 4.0 International license.

B
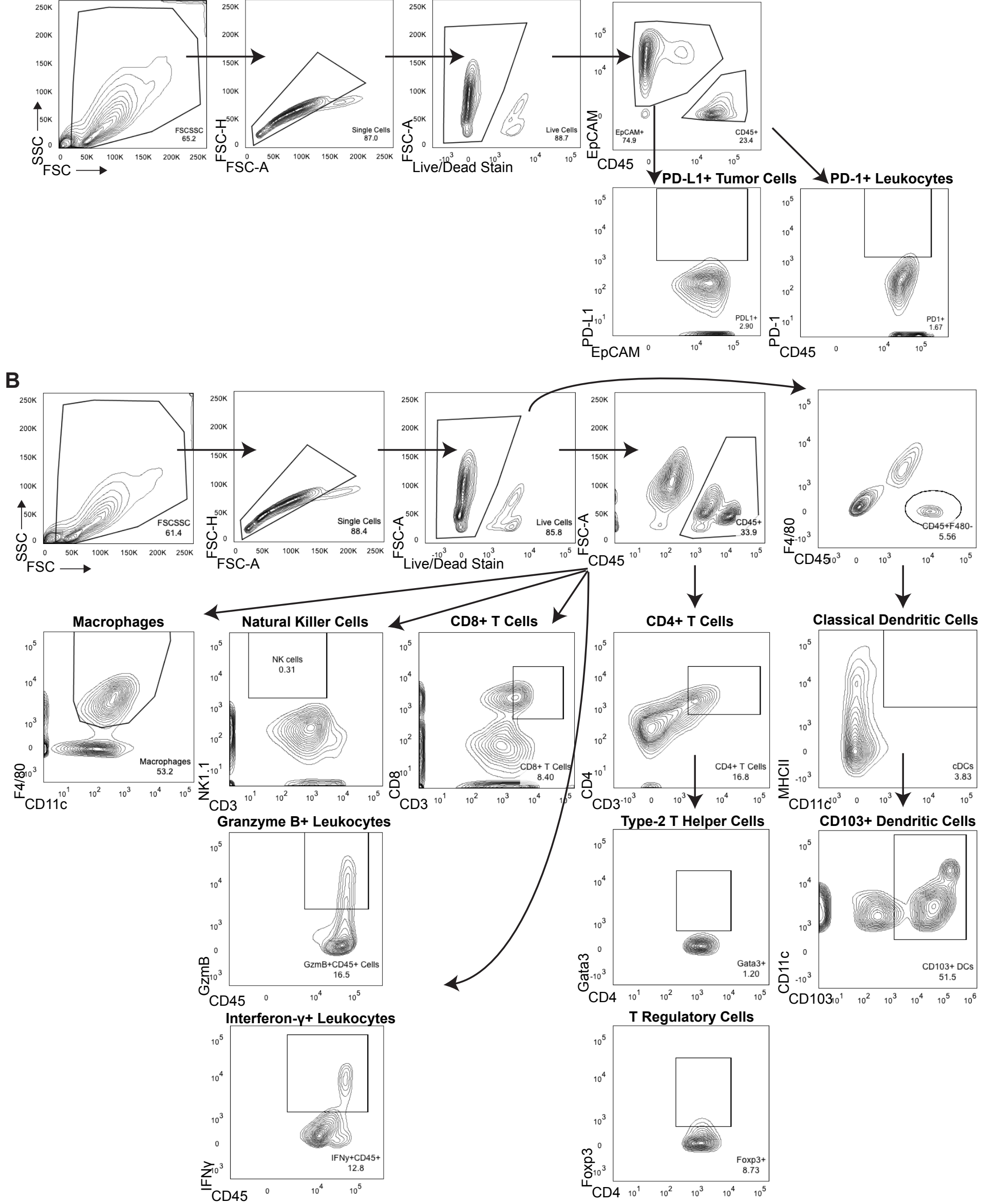

Supplementary Figure S2: Flow cytometry gating strategy. (A) Gating strategy for PD-1 and PD-L1 expression on immune cells and tumor cells. (B) Gating strategy for macrophages, natural killer cells, granzyme B+ immune cells, interferon- $\gamma+$ immune cells, CD8+ T cells, CD4+ T cells, type-2 T helper cells, T regulatory cells, and CD103+ dendritic cells. 


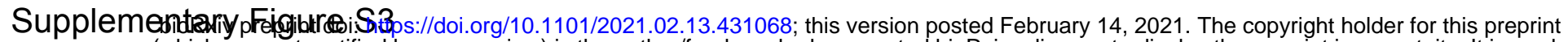
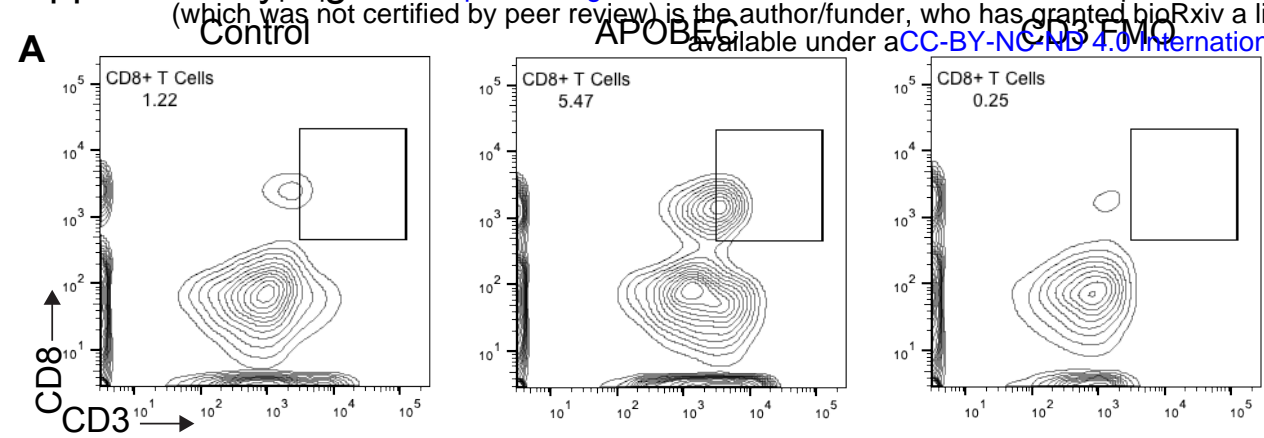

B

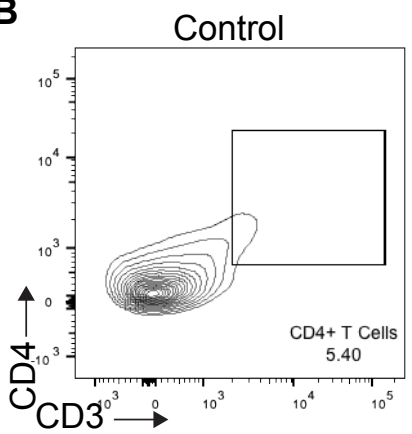

C

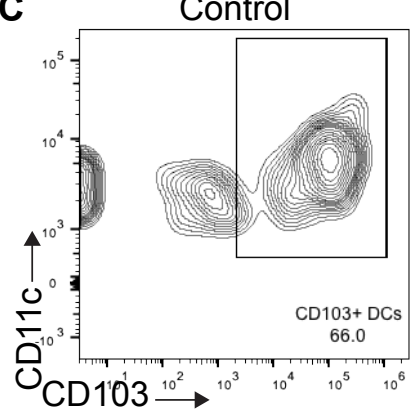

D

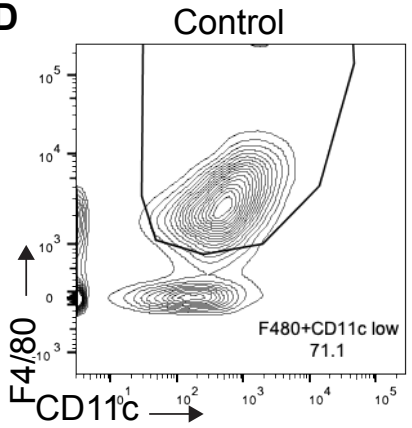

E

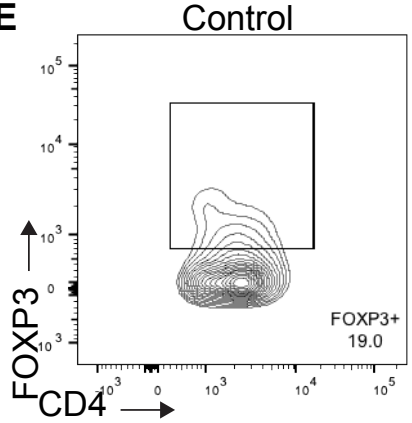

$\mathbf{F}$

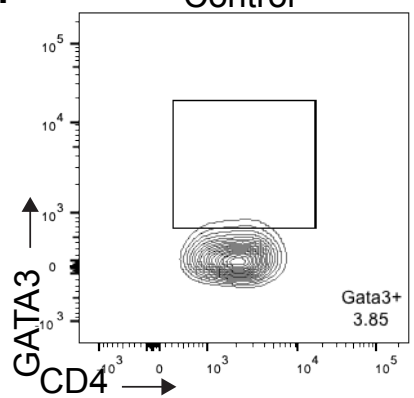

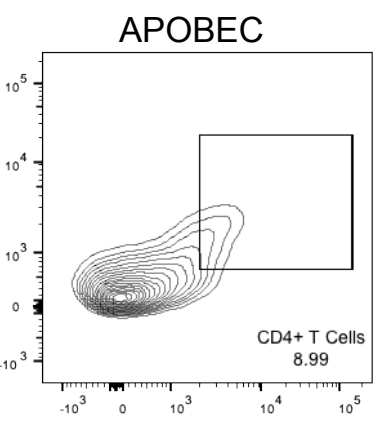

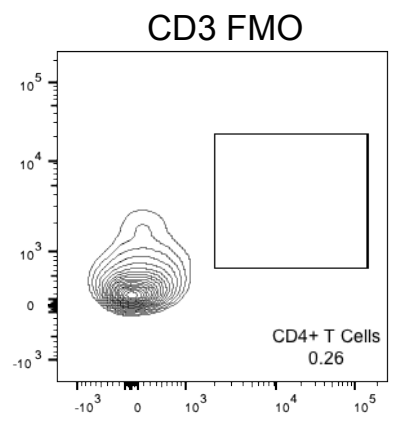

APOBEC
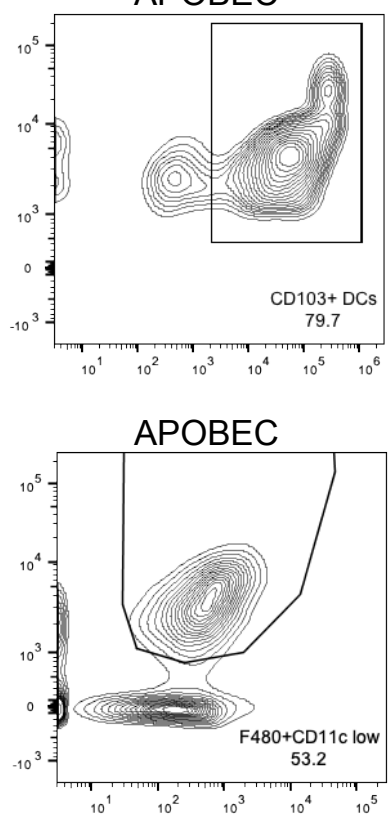

APOBEC

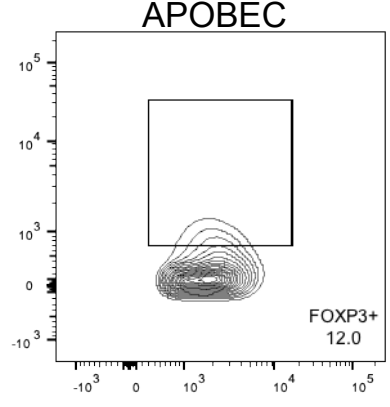

APOBEC

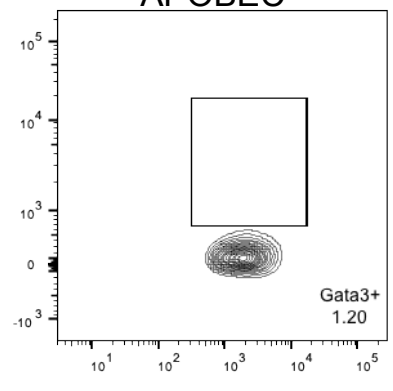

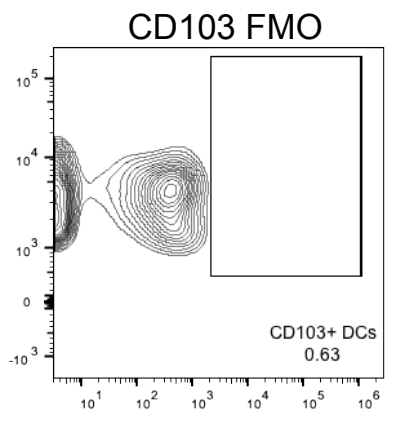
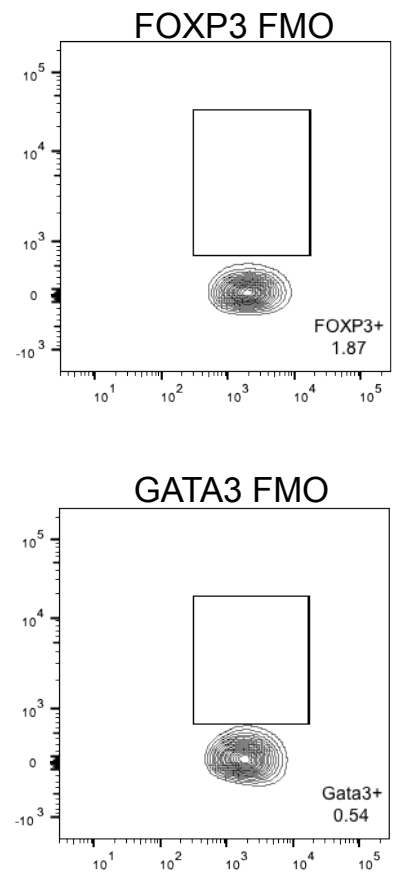

Supplementary Figure S3: Representative FACS plots showing immune cell infiltration in APOBEC tumors. (A) Staining for CD8+ T cells (CD8+CD3+) and CD3 fluorescence minus one (FMO) control without CD3 antibody. (B) Staining for CD4+ T cells (CD4+CD3+) and CD3 FMO. (C) Staining for CD103 expression on dendritic cells (CD103+CD11C+) and CD103 FMO. (D) Staining for macrophages (F4/80 + CD11clow). (E) Staining for $T$ regulatory cells (CD4+FOXP3+) and FOXP3 FMO. (F) Staining for Type-2 $T$ helper cells (CD4+GATA3+) and GATA3 FMO. 
Supplementary Figure S4: Tumor growth inhibition and increased immune infiltration in APOBEC tumors is reproducible and generalizable. (A) Bilateral tumor-draining inguinal lymph nodes (TDLN) were harvested from mice in Figure 2B and aggregated from 4 mice per cohort for flow cytometry. APOBEC TDLNs had increased CD8 + T cells and CD103+ DCs compared to control TDLNs. Error bars denote mean \pm SEM and statistical significance was determined by unpaired Student's t-test. (B) Tumor volume (mm3) over time for unilateral control tumors $(n=5)$, and APOBEC tumors $(n=5)$ generated from SMF-A3B cells in wildtype mice in an independent experiment, demonstrating that the growth defect of $A P O B E C$ tumors is reproducible in an independent experiment. Error bars denote mean \pm SEM. (C) Control tumors $(n=5)$ and APOBEC tumors $(n=5)$ from $(B)$ were harvested and immune profiled by flow cytometry. Quantification shows the APOBEC tumors had increased leukocytes, CD103+ dendritic cells (DCs), and tumor cell PD-L1 expression (MFI, mean fluorescence intensity), while T regulatory cells (Tregs) and type-2 T helper (Th2) cells were reduced in APOBEC tumors. These results demonstrate that immune infiltration in APOBEC tumors is reproducible in an independent experiment. Error bars denote mean \pm SEM and statistical significance was determined by unpaired Student's t-test. (D) qRT-PCR analysis for APOBEC3B expression in EMT6-A3B cultured with or without dox for 2 days. 1,000 ng/mL Dox +/- indicates cells cultured with $1,000 \mathrm{ng} / \mathrm{mL}$ dox for 2 days followed by removal of dox for 3 days prior to analysis. Left: A3B expression relative to $0 \mu \mathrm{g} / \mathrm{mL}$ dox condition. Right: A3B expression relative to BT474 cells. Results show 3 biological replicates and error bars depict mean \pm SEM. Significance was determined using a one-way ANOVA and Tukey's multiple comparisons test. (E) EMT6-A3B cells were cultured as in (D) and cell lysates were harvested for western blot of HA-tagged A3B protein. (F) EMT6-A3B cells were cultured as in (D) and cell lysates harvested for in vitro deaminase activity assay. Deaminase activity is comparable to that of human cell line, BT474. SKBR3 human cell line is A3B-null and shown as a negative control. (G) Tumor volume curves for control (-dox; $n=16)$ and APOBEC (+dox; $n=16)$ tumors derived from EMT-A3B cells orthotopically implanted in the mammary gland of syngeneic $\mathrm{BALB} / \mathrm{c}$ mice. Error bars denote mean \pm SEM and statistical significance was determined by two-way repeatedmeasures ANOVA. (H) The fraction of control and APOBEC EMT6 tumors that grew or spontaneously regressed following tumor cell injection. Fisher's exact test, $p=0.0659$. (I) Flow cytometry quantification of leukocytes in control $(n=6)$ and APOBEC $(n=6)$ EMT6 tumors from $(G)$. Error bars denote mean \pm SEM and statistical significance was determined by unpaired Student's t-test. ${ }^{*} p<0.05,{ }^{* *} p<0.01,{ }^{* * *} p<0.0001$ 
Supplementary Figure S5: The APOBEC tumor growth defect requires the catalytic activity of A3B. (A) qRTPCR of A3B gene expression in SMF-A3B ${ }^{\text {inactive }}$ cells treated with $1 \mathrm{ug} / \mathrm{mL}$ dox for 5 days. Error bars denote mean \pm SD for 3 technical replicates and statistical significance was determined by unpaired Student's t-test. (B)

Immunofluorescence staining for HA epitope-tagged A3B in control tumors (-dox) and tumors expressing A3Binactive (+dox). (C) In vitro deaminase activity assay in SMF-A3B ${ }^{\text {inactive }}$ cells treated with dox. SMF-A3B cells are shown as a control. (D) Tumor volume $(\mathrm{mm} 3)$ over time for control tumors (-dox; $n=14)$ and tumors expressing $A 3 B^{\text {inactive }}$ $(+$ dox $; n=14)$ in wildtype mice. Error bars denote mean \pm SEM. Statistical significance was determined by two-way repeated-measures ANOVA. (E) Quantification of IHC staining for CD45 (left) or CD3 (right) in control tumors ( $n=5$ ) and tumors expressing $A 3 B^{\text {inactive }}(n=5)$. Four fields of view were quantified for each tumor. Error bars denote mean \pm SD. Statistical significance was determined by unpaired Student's t-test. (F) Growth curves for control and in vitro APOBEC mutagenized cells. Data are shown as mean \pm SD of 4 replicates. (G) Schematic showing experimental design for tumor growth experiment. SMF-A3B cells were cultured with or without dox for 2 weeks, then dox was removed for 2 weeks. These in vitro APOBEC mutagenized cells or control cells were orthotopically implanted in the mammary gland of mice in the absence of dox. $(\mathbf{H})$ Tumor volume $(\mathrm{mm} 3)$ over time for control $(n=14)$ and in vitro APOBEC mutagenized tumors $(n=14)$ in wildtype mice. Error bars denote mean \pm SEM. Statistical significance was determined by two-way repeated-measures ANOVA. (I) qRT-PCR analysis for Granzyme A (Gzma), Perforin-1 (Prf-1), and T-bet (Tbx21) in control $(n=6)$ and in vitro APOBEC mutagenized tumors $(n=6)$. All genes showed a trend toward increased expression in the in vitro APOBEC mutagenized cohort that did not reach statistical significance. (J) Tumor volume $(\mathrm{mm} 3)$ over time for control $(\mathrm{n}=16)$ and in vitro APOBEC mutagenized tumors $(n=16)$ in NSG mice. Error bars denote mean \pm SEM. Statistical significance was determined by two-way repeatedmeasures ANOVA and Tukey's multiple comparisons test. Note that control mice are the same as in S1A. ns $p>$ $0.05,{ }^{* *} p<0.01,{ }^{* * *} p<0.001$ 


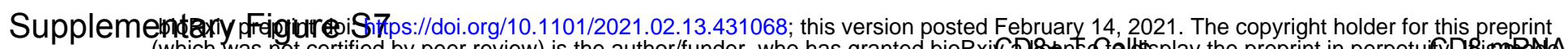

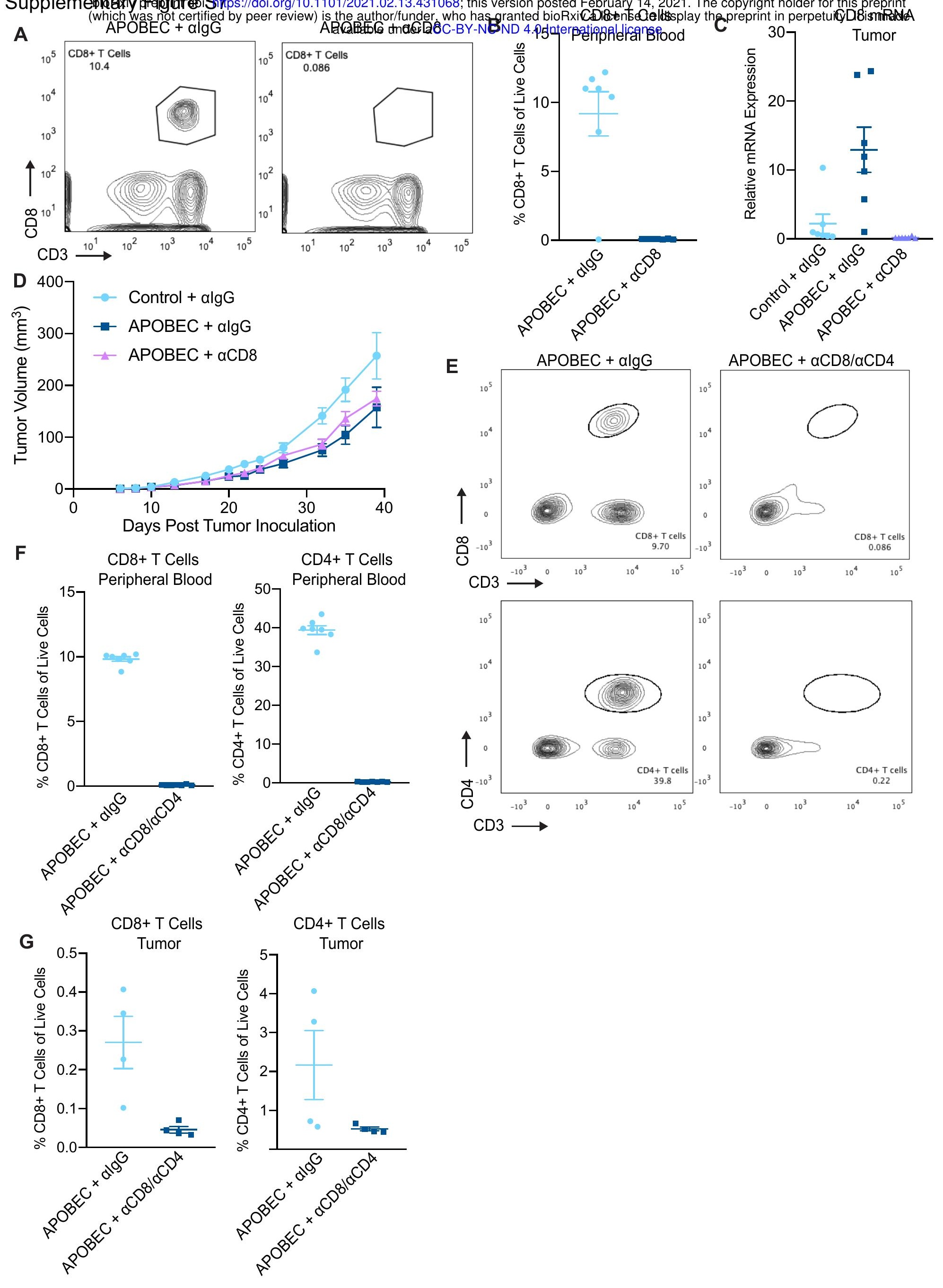


bioRxiv preprint doi: https://doi.org/10.1101/2021.02.13.431068; this version posted February 14, 2021. The copyright holder for this preprint (which was not certified by peer review) is the author/funder, who has granted bioRxiv a license to display the preprint in perpetuity. It is made Supplementary Figure S7: CD4+/CD8+ Tole under aCC-BY-NC-ND 4.0 International license. defect of APOBEC tumors. (A) Flow cytometry showing depletion of CD8+ T cells in peripheral blood following intraperitoneal injection of an in vivo CD8 depleting antibody (300 $\mu \mathrm{g} / \mathrm{dose})$ or isotype-control antibody twice weekly. Peripheral blood was assayed on day 9 post tumor inoculation. (B) Quantification of CD8+ T cells in peripheral blood of isotype-control antibody treated mice $(n=7)$ and $\alpha C D 8$ antibody treated mice $(n=7)$ as in $(A)$. Error bars denote mean \pm SEM. (C) qRT-PCR for CD8 expression in tumors from the indicated cohorts: control tumors + algG $(n=7)$; APOBEC tumors + algG $(n=7)$; APOBEC tumors + $\alpha$ CD8 $(n=7)$. Error bars denote mean \pm SEM. (D) Tumor volume (mm3) over time for control + algG $(n=14)$, APOBEC + algG $(n=14)$, and APOBEC + $\alpha C D 8(n=14)$ tumors in wildtype mice. Error bars denote mean \pm SEM. (E) Flow cytometry showing depletion of CD8+ and CD4+ T cells in peripheral blood following intraperitoneal injection of CD8 and CD4 depleting antibodies $(200 \mu \mathrm{g}$ CD8 and $200 \mu \mathrm{g}$ CD4/dose) or isotype-control antibody twice weekly. Peripheral blood was assayed on day 25 post tumor inoculation. (F) Quantification of CD4+ and CD8+ T cells in peripheral blood of isotype-control antibody treated mice $(n=7)$ and $\alpha C D 8 / a C D 4$ antibody treated mice $(n=7)$ as in $(E)$. Error bars denote mean \pm SEM. (G) Flow cytometry quantification of CD8+ and CD4+ T cells in APOBEC tumors treated with isotype-control antibody or aCD8/aCD4 depleting antibodies. Error bars denote mean \pm SEM. 


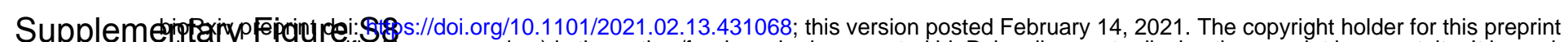
(which was not certified by peer review) is the author/funder, who has granted bioRxiv a license to display the preprint in perpetuity. It is made A PD-1/CTLA-4 Combination available under aCC-BY-NCAD 4.0 Internatioßat liqeperonotherapy

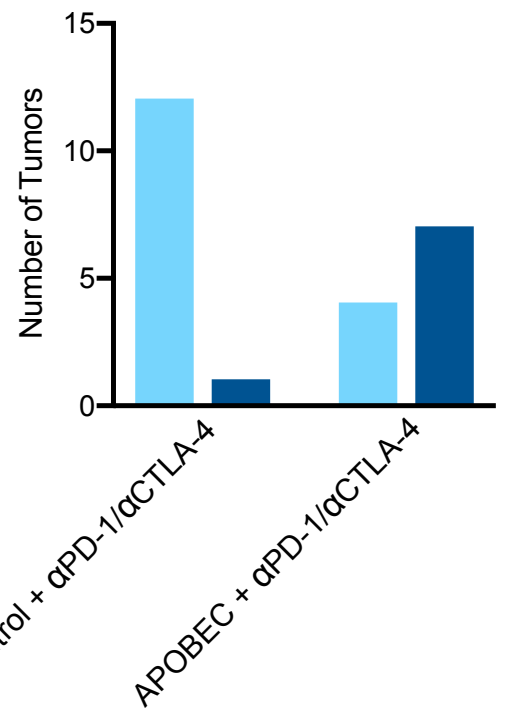

\section{No Response \\ CR/PR}

Fisher's exact test $p=0.0078$

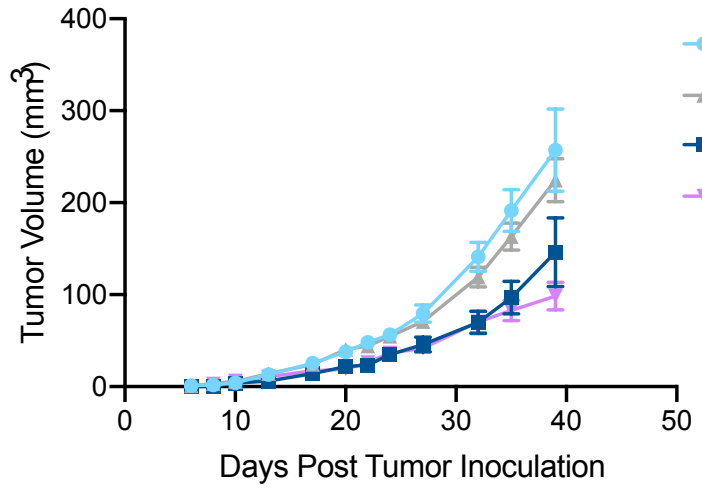

MTM

6 doses of aPD-1

Supplementary Figure S8: APOBEC activity renders HER2-driven mammary tumors responsive to combination anti-PD-1/anti-CTLA-4 therapy, but not anti-PD-1 monotherapy. (A) Response of control and APOBEC tumors to combination PD-1/CTLA-4 therapy. Number of tumors with no response or complete response/ partial response (CR/PR) are depicted. Statistical significance was determined by Fisher's exact test $(p=0.0078)$. $(B)$ Tumor volume (mm3) over time for Control + algG ( $n=14)$, Control + $\alpha P D-1(n=14)$, APOBEC + algG $(n=14)$, and APOBEC + aPD-1 $(n=14)$ tumors in wildtype mice. Mice were administered 6 doses of $200 \mu g$ of PD-1 or lgG isotype antibody on day $13,15,17,20,22,24$ post-tumor inoculation. Error bars denote mean \pm SEM. 
A

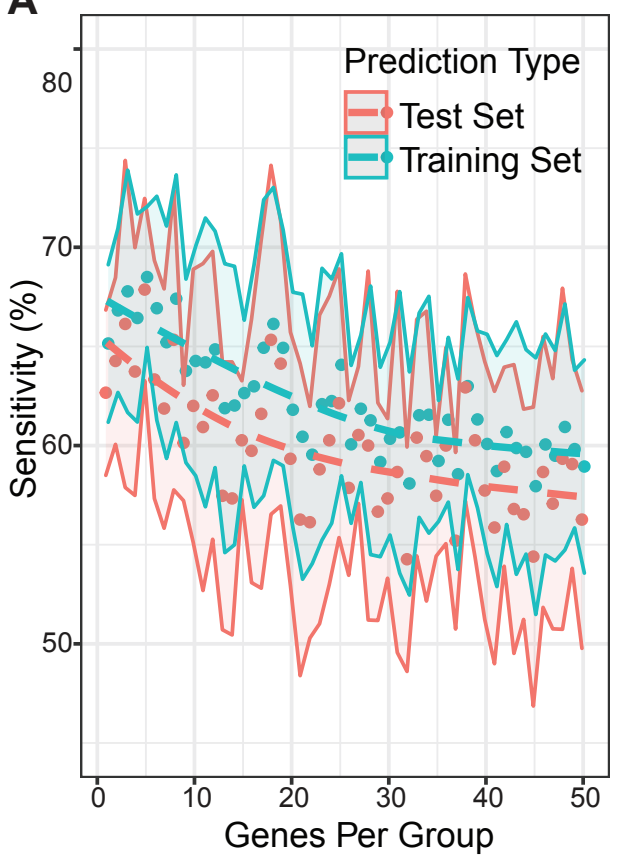

C

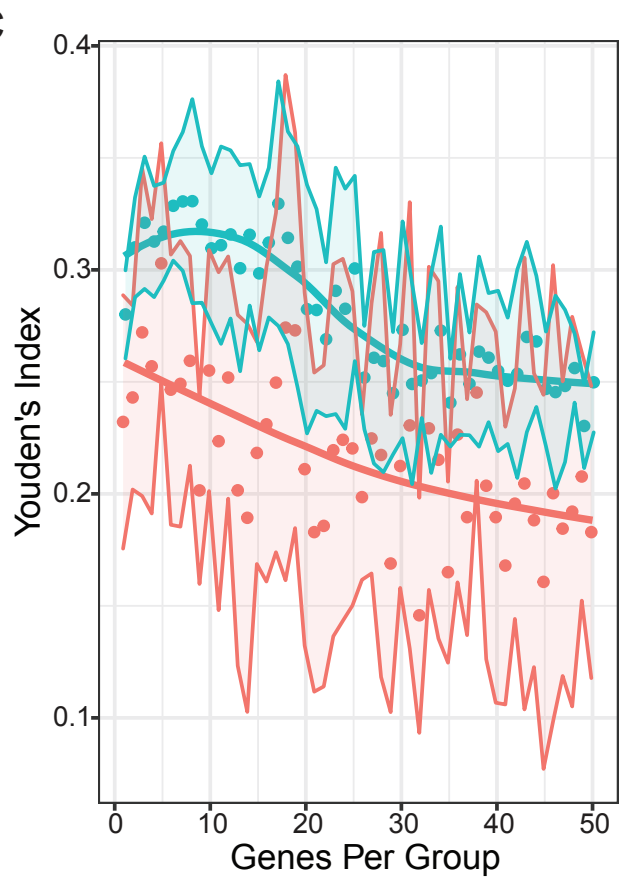

B

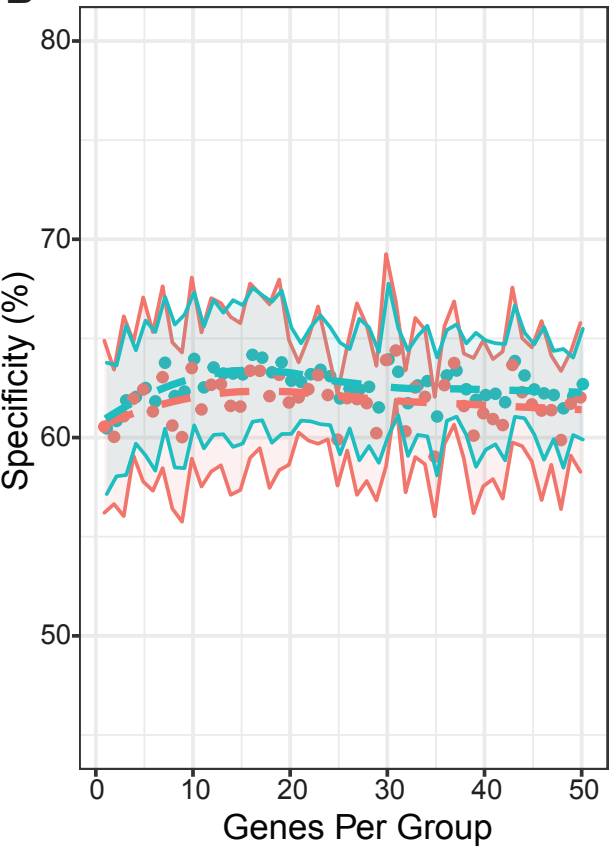

D

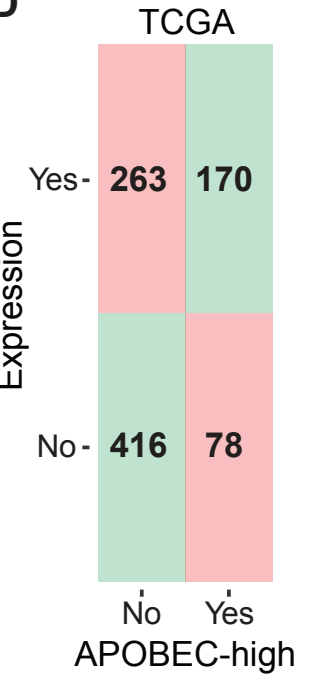

Supplementary Figure S10: Methods for gene expressed-based classifier of APOBEC mutational signatures. (A-B) Ten-fold cross validated sensitivity (A) and specificity (B) of TCGA classification to nearest centroids (ClaNC) predictor using 1 to 50 of the top $5 \%$ most variably expressed genes to predict high versus low APOBEC enrichment by WES. In each run of the cross validation, $90 \%$ of all tumors were randomly selected to serve as the training set and the remaining $10 \%$ served as the test set. Points represent the mean sensitivity and specificity across the 10 folds, and confidence bands show standard deviation. (C) Ten-fold cross validated Youden's index (sensitivity + specificity - 1) for test and training sets. The maximum Youden's index in the test set was reached using 5 genes per group (10 total) and was therefore selected for the final model. Points represent mean Youden's index, and confidence bands show standard deviation. (D) Confusion matrix of predicted (gene expression classifier) versus true (APOBEC enrichment score $>2$ by WES) classifications in the full TCGA dataset using the 10 gene predictor. Squares in red (upper left and bottom right) denote incorrect classifications and squares in green (upper right and bottom left) represent correct classifications. 